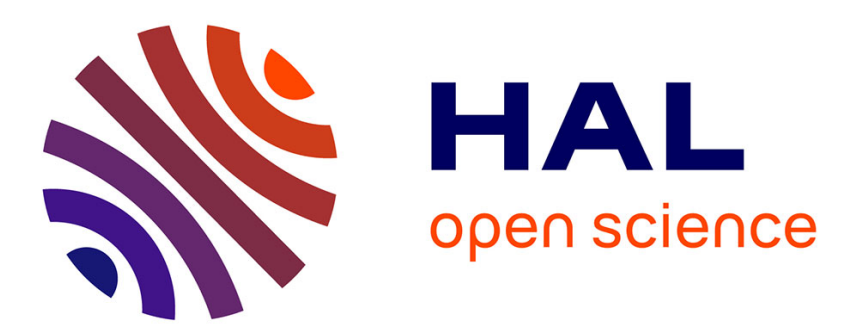

\title{
A multiscale method with patch for the solution of stochastic partial differential equations with localized uncertainties
}

\author{
Mathilde Chevreuil, Anthony Nouy, Elias Safatly
}

\section{To cite this version:}

Mathilde Chevreuil, Anthony Nouy, Elias Safatly. A multiscale method with patch for the solution of stochastic partial differential equations with localized uncertainties. Computer Methods in Applied Mechanics and Engineering, 2013, 255, pp.255-274. 10.1016/j.cma.2012.12.003 . hal-00733739

\section{HAL Id: hal-00733739 \\ https://hal.science/hal-00733739}

Submitted on 19 Sep 2012

HAL is a multi-disciplinary open access archive for the deposit and dissemination of scientific research documents, whether they are published or not. The documents may come from teaching and research institutions in France or abroad, or from public or private research centers.
L'archive ouverte pluridisciplinaire $\mathbf{H A L}$, est destinée au dépôt et à la diffusion de documents scientifiques de niveau recherche, publiés ou non, émanant des établissements d'enseignement et de recherche français ou étrangers, des laboratoires publics ou privés. 


\title{
A multiscale method with patch for the solution of stochastic partial differential equations with localized uncertainties
}

\author{
M. Chevreuil, A. Nouy, E. Safatly \\ LUNAM Université, Ecole Centrale Nantes, Université de Nantes, GeM, UMR CNRS \\ 6183, 1 rue de la Noë, BP 92101, 44321 Nantes Cedex 3, France.
}

\begin{abstract}
We here propose a multiscale numerical method for the solution of stochastic partial differential equations with localized uncertainties. It is based on a multiscale domain decomposition method that exploits the localized side of uncertainties and incidentally improves the conditioning of the problem by operating a separation of scales. An efficient iterative algorithm is proposed that requires the solution of a sequence of simple global problems at a macro scale, involving a deterministic operator, and local problems at a micro scale for which we have the possibility to use fine approximation spaces. Global and local problems are solved using tensor approximation methods allowing the representation of high dimensional stochastic parametric solutions. Convergence properties of these tensor based methods, which are closely related to spectral decompositions, benefit from the separation of scales. Different types of uncertainties are considered at the micro level. They may be associated with some variability in the operator or source terms, or even with some geometrical variability. In the latter case, specific reformulations of local problems using fictitious domain methods are introduced.

Key words: Uncertainty quantification; Multiscale stochastic PDE; Numerical zoom; Domain Decomposition; Tensor approximation; High dimensional problems.
\end{abstract}

\section{Introduction}

Uncertainty quantification methods using functional approaches have emerged for the last two decades in computational engineering. Numerous developments have been realized for the propagation using functional approaches (see reviews $[1,2,3,4])$. Recent works have been devoted to the propagation of uncertainties through stochastic multiscale models using these functional approaches. In

Email address: mathilde.chevreuil@univ-nantes.fr (M. Chevreuil)

Preprint submitted to Elsevier

August 16, 2012 
the case of random heterogeneous media allowing a separation of scales, upscaling or homogenenization methods have been introduced, see for instance $[5,6,7,8,9]$. However the propagation of uncertainties through multiscale stochastic models remains today a challenging issue for they give rise to high dimensional stochastic problems and this high dimensionality is thus to be handled genuinely. Moreover, monoscale numerical approaches clearly suffer from the complexity of multiscale solutions that present very high spectral content.

In the present work, we focus on multiscale problems with localized uncertainties (in medium property, source terms or geometry). In the presence of numerous localized sources of uncertainties, dedicated approaches have to be developed in order to handle the high dimensionality and complexity of associated multiscale models. At the deterministic level, dedicated methods have met the demand of coupling numerical models at different scales and some have been extended to stochastic models. Among these deterministic methods, one can distinguish the mono-model methods based on adaptive mesh or enrichment techniques $[10,11,12,13]$ from the multi-model methods based on patches as the global-local iterative methods proposed in $[14,15,16,17,18]$ or the bridging methods proposed in $[19,20,21]$ or in [22] with the Arlequin method. The latter has been exploited in the stochastic framework for deterministic-stochastic coupling in $[23,24]$ for a homogenization purpose.

We here propose a dedicated method based on a multiscale domain decomposition method that exploits the localized side of uncertainties. It belongs to the class of the global-local iterative methods mentionned above. In its extension to the stochastic framework, an efficient iterative algorithm is proposed that requires the solution of a sequence of simple global problems at a macro scale, involving a deterministic operator, and local problems at a micro scale for which we have the possibility to use fine approximation spaces. In the meanwhile the separation of scales has the advantage of improving the conditioning of the problem. In order to address the high dimensionality that arises from these multiscale problems with numerous sources of uncertainties, the global and local problems are solved using tensor based approximation methods allowing the representation of high dimensional stochastic parametric solutions. Convergence properties of these tensor based methods, which are closely related to spectral decompositions, benefit from the separation of scales. Different formats of tensor representations can be expoited [25]. Here the classical canonical decompositions and the hierarchical canonical decompositions as in $[26,27]$ are introduced, the latter ones giving very low ranks representations.

Different types of uncertainties are considered at the micro level. They may be associated with some variability in the operator or source terms, or even with some geometrical uncertainty. In the latter case, specific reformulations of local problems using fictitious domain methods are introduced in order to formulate the problem on a tensor product space $[28,29,30]$.

The paper is structured as follows. In section 2, the model problem with localized variabilities is first presented. Then the global-local iterative algorithm is introduced in section 3. Section 4 is dedicated to the approximate solutions of the global and local problems involved in the iterative algorithm: definition of 
approximation spaces and fictitious domain methods for the reformulation of the local problems when these present geometrical variabilities. Section 5 extends the method to the case of multiple patches with independent variabilities. The behavior of the global-local iterative algorithm is analysed in section 6 on a first numerical example with four patches and with no geometrical details. The convergence and robustness results of the global-local iterative algorithm proven in section 3 are notably illustrated on this example. The influence of the sizes of the patches on the convergence of the algorithm is also analysed. Tensor approximation methods are finally introduced in section 7 for the solution of local and global problems in order to handle the high dimensionality. They are applied in section 8 to a high dimensional problem which contains geometrical variabilities. This last illustration shows the relevance of the use of tensor approximation methods and in particular of hierarchical decompositions.

\section{Problem with localized variabilities}

We consider a diffusion problem defined on a domain $\Omega \subset \mathbb{R}^{d}$ :

$$
\begin{aligned}
& -\nabla \cdot(K \nabla u)=f \quad \text { on } \Omega, \\
& K \nabla u \cdot n=0 \text { on } \Gamma_{N}, \\
& u=0 \text { on } \Gamma_{D},
\end{aligned}
$$

with $K$ a diffusion parameter, and $\Gamma_{D}$ and $\Gamma_{N}$ the Dirichlet and Neumann boundaries respectively. We denote by $\xi$ a set of random parameters, with values in $\Xi$, modeling the uncertainties on the geometry, the source term and the diffusion coefficient. We denote by $\left(\Xi, \mathcal{B}, P_{\xi}\right)$ the associated probability space, where $P_{\xi}$ is the probability law of $\xi$.

\subsection{Function spaces}

For a Hilbert space $\mathcal{H}$ equipped with an inner product norm $|\cdot|$, we denote by $\mathcal{H}^{\Xi}$ the Bochner space of square integrable functions defined on the measure space $\left(\Xi, \mathcal{B}, P_{\xi}\right)$ and with values in $\mathcal{H}$ :

$$
\mathcal{H}^{\Xi}=L_{P_{\xi}}^{2}(\Xi ; \mathcal{H})=\left\{v: y \in \Xi \mapsto v(y) \in \mathcal{H} ; \mathbb{E}\left(|v(\xi)|^{2}\right)<+\infty\right\},
$$

where $\mathbb{E}(\cdot)$ is the mathematical expectation defined by

$$
\mathbb{E}(v)=\int_{\Xi} v(y) d P_{\xi}(y) .
$$

Bochner space $\mathcal{H}^{\Xi}$ is a Hilbert space when equipped with the following inner product norm

$$
\|v\|=\mathbb{E}\left(|v(\xi)|^{2}\right)^{1 / 2}
$$

For $\mathcal{H}=\mathbb{R}$, we use the notations $\mathcal{S}:=\mathbb{R}^{\Xi}=L_{P_{\xi}}^{2}(\Xi ; \mathbb{R}):=L_{P_{\xi}}^{2}(\Xi)$. Note that $\mathcal{H}$ can be a random function space, i.e. dependent on $\xi$ (e.g. when considering a space of functions defined on an uncertain domain). In the case where $\mathcal{H}$ is 
deterministic, the Bochner space can be identified with the tensor Hilbert space $\mathcal{H} \otimes \mathcal{S}:$

$$
\mathcal{H}^{\Xi}=L_{P_{\xi}}^{2}(\Xi ; \mathcal{H}) \simeq \mathcal{H} \otimes \mathcal{S} .
$$

\subsection{Initial weak formulation of the problem}

Let introduce the Hilbert space $\mathcal{V}=\left\{v \in H^{1}(\Omega) ; v=0\right.$ on $\left.\Gamma_{D}\right\}$ equipped with the inner product norm $|u|_{\Omega}=\left(\int_{\Omega} \nabla u \cdot \nabla u\right)^{1 / 2}$. Let $\mathcal{V}^{\Xi}=L_{P_{\xi}}^{2}(\Xi ; \mathcal{V})$ be the Hilbert space equipped with the norm $\|\cdot\|_{\Omega}=\mathbb{E}\left(|\cdot|_{\Omega}^{2}\right)^{1 / 2}$. We introduce the classical weak formulation of problem (1):

$$
u \in \mathcal{V}^{\Xi}, \quad a_{\Omega}(u, \delta u)=\ell_{\Omega}(\delta u) \quad \forall \delta u \in \mathcal{V}^{\Xi},
$$

with

$$
\begin{aligned}
& a_{\Omega}(u, \delta u)=\mathbb{E}\left(\int_{\Omega} K \nabla u \cdot \nabla \delta u\right)=\int_{\Xi} \int_{\Omega} K \nabla u \cdot \nabla \delta u d P_{\xi}, \\
& \ell_{\Omega}(\delta u)=\mathbb{E}\left(\int_{\Omega} f \delta u\right)=\int_{\Xi} \int_{\Omega} f \delta u d P_{\xi} .
\end{aligned}
$$

We introduce the notation $\Omega * \Xi=\left\{(x, y) \in \mathbb{R}^{d} \times \Xi ; x \in \Omega(y)\right\}$. Note that in the case of a deterministic domain $\Omega$, we simply have $\Omega * \Xi=\Omega \times \Xi$. Problem (2) is well-posed if $f \in L^{2}(\Omega)^{\Xi}$ and if $K$ is uniformly bounded and elliptic on $\Omega * \Xi$, i.e. there exist constants $K_{i n f}>0$ and $K^{\text {sup }}>0$ such that we have almost everywhere on $\Omega * \Xi$

$$
K_{\text {inf }}|\zeta|^{2} \leq \zeta \cdot K(x, y) \zeta \leq K^{\text {sup }}|\zeta|^{2} \quad \forall \zeta \in \mathbb{R}^{d} .
$$

\subsection{Patch containing localized variabilities}

We consider that the diffusion coefficient $K$, the source term $f$ or the domain $\Omega$ are uncertain only on a part $\Lambda \subset \Omega$. $\Lambda$ is called a patch. The boundary $\partial \Lambda$ of this patch contains the eventual uncertain part of the boundary $\partial \Omega$. That means that $\Lambda$ eventually depends on $\xi$ and is such that

$$
\Omega(\xi)=(\Omega \backslash \Lambda) \cup \Lambda(\xi),
$$

with $\Omega \backslash \Lambda$ deterministic. We denote by $\Gamma=\partial(\Omega \backslash \Lambda) \cap \partial \Lambda$ the deterministic interface between $\Omega \backslash \Lambda$ and the patch $\Lambda$ (see figure 1). We then consider that

$$
K(x, \xi)=\left\{\begin{array}{ll}
K_{0}(x) & \text { for } x \in \Omega \backslash \Lambda \\
K(x, \xi) & \text { for } x \in \Lambda(\xi)
\end{array} \text { and } f(x, \xi)= \begin{cases}f_{0}(x) & \text { for } x \in \Omega \backslash \Lambda \\
f(x, \xi) & \text { for } x \in \Lambda(\xi)\end{cases}\right.
$$




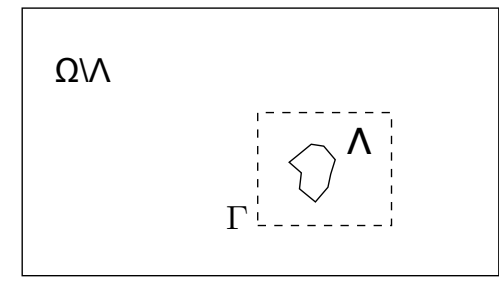

Figure 1: Patch $\Lambda \subset \Omega$ containing localized uncertainties and interface $\Gamma=\partial(\Omega \backslash \Lambda) \cap \partial \Lambda$.

\subsection{Domain decomposition}

We introduce the following splitting of the solution $u \in \mathcal{V}^{\Xi}$ :

$$
u=\left\{\begin{array}{lll}
U & \text { on } & \Omega \backslash \Lambda \\
w & \text { on } & \Lambda
\end{array},\right.
$$

with the following continuity conditions on $\Gamma$ interpreted in a weak sense:

$$
w=U \text { and } \nabla U \cdot n=\nabla w \cdot n \text { on } \Gamma,
$$

where $n$ denotes the unit outward normal to $\partial \Lambda$. We consider that $\Gamma \cap \Gamma_{D}=\emptyset$ and we split the Dirichlet boundary $\Gamma_{D}$ into the Dirichlet boundary of the patch $\Gamma_{D}^{\Lambda}=\Gamma_{D} \cap \partial \Lambda$ (eventual internal boundary of the patch) and the complementary part $\Gamma_{D}^{\Omega \backslash \Lambda}=\Gamma_{D} \backslash \Gamma_{D}^{\Lambda}$. We then introduce the following spaces

$$
\begin{aligned}
& \mathcal{U}=\left\{U \in H^{1}(\Omega \backslash \Lambda) ; U=0 \text { on } \Gamma_{D}^{\Omega \backslash \Lambda}\right\} \\
& \mathcal{W}=\left\{w \in H^{1}(\Lambda) ; w=0 \text { on } \Gamma_{D}^{\Lambda}\right\} \\
& \mathcal{M}=H^{-1 / 2}(\Gamma)
\end{aligned}
$$

and the associated Bochner spaces $\mathcal{U}^{\Xi}, \mathcal{W}^{\Xi}$ and $\mathcal{M}^{\Xi}$.

Problem (2) is then equivalent to finding $(U, w, \lambda) \in \mathcal{U}^{\Xi} \times \mathcal{W}^{\Xi} \times \mathcal{M}^{\Xi}$ such that for all $(\delta U, \delta w, \delta \lambda) \in \mathcal{U}^{\Xi} \times \mathcal{W}^{\Xi} \times \mathcal{M}^{\Xi}$,

$$
\left\{\begin{aligned}
a_{\Omega \backslash \Lambda}(U, \delta U)+b_{\Gamma}(\lambda, \delta U) & =\ell_{\Omega \backslash \Lambda}(\delta U) \\
a_{\Lambda}(w, \delta w)-b_{\Gamma}(\lambda, \delta w) & =\ell_{\Lambda}(\delta w) \\
b_{\Gamma}(\delta \lambda, U-w) & =0
\end{aligned}\right.
$$

where for some subdomain $\mathcal{O} \subset \Omega$,

$$
a_{\mathcal{O}}(v, \delta v)=\mathbb{E}\left(\int_{\mathcal{O}} K \nabla v \cdot \nabla \delta v\right), \quad \ell_{\mathcal{O}}(v)=\mathbb{E}\left(\int_{\mathcal{O}} f v\right)
$$

and where

$$
b_{\Gamma}(\lambda, v)=\mathbb{E}\left(\int_{\Gamma} \lambda v\right) .
$$

$\lambda$ represents a Lagrange multiplier ensuring the weak continuity of $u$ on the interface $\Gamma$. 


\section{A global-local iterative algorithm}

\subsection{Domain decomposition with overlapping domains}

In the case where $\Lambda$ contains geometrical details, i.e. if $\partial \Lambda \backslash \Gamma \neq \emptyset$, we introduce a fictitious patch $\widetilde{\Lambda} \supset \Lambda$ such that $\partial \widetilde{\Lambda}=\Gamma$, which means that $\widetilde{\Lambda}$ is deterministic and does not contain any internal boundaries, i.e. no geometrical details (see figure 2). We then denote by

$$
\widetilde{\Omega}=(\Omega \backslash \Lambda) \cup \widetilde{\Lambda},
$$

which is a deterministic fictitious domain containing $\Omega$ and such that $\widetilde{\Omega} \backslash \widetilde{\Lambda}=$ $\Omega \backslash \Lambda$.

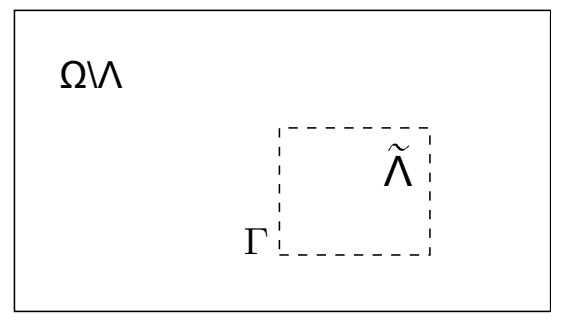

Figure 2: Fictitious patch $\widetilde{\Lambda}$ such that $\partial \widetilde{\Lambda}=\Gamma$.

We now consider a prolongation of function $U$ from $\Omega \backslash \Lambda$ to $\widetilde{\Omega}$. We introduce on $\widetilde{\Omega}$ a fictitious diffusion field $\widetilde{K}$ such that

$$
\widetilde{K}=K \quad \text { on } \quad \Omega \backslash \Lambda,
$$

and such that it verifies almost everywhere on $\widetilde{\Omega} \times \Xi$

$$
\tilde{K}_{i n f}|\zeta|^{2} \leq \zeta \cdot \widetilde{K}(x, y) \zeta \leq \tilde{K}^{\text {sup }}|\zeta|^{2} \quad \forall \zeta \in \mathbb{R}^{d},
$$

for some constants $\tilde{K}^{\text {sup }} \geq \tilde{K}_{\text {inf }}>0$. We then introduce the new function space $\tilde{\mathcal{U}}^{\Xi}$ with

$$
\widetilde{\mathcal{U}}=\left\{U \in H^{1}(\widetilde{\Omega}) ; U=0 \text { on } \Gamma_{D}^{\Omega \backslash \Lambda}\right\} .
$$

For a subset $\mathcal{O} \subset \widetilde{\Omega}$, we define a bilinear form $c_{\mathcal{O}}: \widetilde{\mathcal{U}}^{\Xi} \times \widetilde{\mathcal{U}}^{\Xi} \rightarrow \mathbb{R}$ associated with the fictitious diffusion coefficient:

$$
c_{\mathcal{O}}(U, \delta U)=\mathbb{E}\left(\int_{\mathcal{O}} \widetilde{K} \nabla U \cdot \nabla \delta U\right) .
$$

We notice that by condition (6) on $\widetilde{K}$, we have

$$
a_{\Omega \backslash \Lambda}(U, \delta U)=c_{\Omega \backslash \Lambda}(U, \delta U)=c_{\widetilde{\Omega}}(U, \delta U)-c_{\widetilde{\Lambda}}(U, \delta U) .
$$


Problem (4) can now be reformulated as follows: find $(U, w, \lambda) \in \widetilde{\mathcal{U}}^{\Xi} \times \mathcal{W}^{\Xi} \times \mathcal{M}^{\Xi}$ such that for all $(\delta U, \delta w, \delta \lambda) \in \widetilde{\mathcal{U}}^{\Xi} \times \mathcal{W}^{\Xi} \times \mathcal{M}^{\Xi}$,

$$
\left\{\begin{aligned}
c_{\widetilde{\Omega}}(U, \delta U)-c_{\widetilde{\Lambda}}(U, \delta U)+b_{\Gamma}(\lambda, \delta U) & =\ell_{\Omega \backslash \Lambda}(\delta U) \\
a_{\Lambda}(w, \delta w)-b_{\Gamma}(\lambda, \delta w) & =\ell_{\Lambda}(\delta w) \\
b_{\Gamma}(\delta \lambda, U-w) & =0
\end{aligned}\right.
$$

Note that problem (9) does not define the prolongation $U$ inside the fictitious patch $\widetilde{\Lambda}$. In other words, formulated on $\widetilde{\mathcal{U}}^{\Xi} \times \mathcal{W}^{\Xi} \times \mathcal{M}^{\Xi}$, this problem admits an infinite number of solutions. As we will see, a particular prolongation will arise from the definition of the following algorithm, which corresponds to considering the formulation (9) in a suitable subspace of $\widetilde{\mathcal{U}}^{\Xi} \times \mathcal{W}^{\Xi} \times \mathcal{M}^{\Xi}$.

\subsection{A global-local algorithm}

We now introduce the following iterative algorithm that defines a sequence $\left\{\left(U_{n}, w_{n}, \lambda_{n}\right)\right\}_{n \geq 0}$. We start with $w_{0}=0, \lambda_{0}=0$ and $U_{0}=0$ and for $n \geq 1$, we define $\left(U_{n}, w_{n}, \lambda_{n}\right)$ as follows.

Global step. We compute $\hat{U}_{n} \in \tilde{\mathcal{U}}^{\Xi}$ such that for all $\delta U \in \tilde{\mathcal{U}}^{\Xi}$,

$$
c_{\widetilde{\Omega}}\left(\hat{U}_{n}, \delta U\right)=c_{\widetilde{\Lambda}}\left(U_{n-1}, \delta U\right)-b_{\Gamma}\left(\lambda_{n-1}, \delta U\right)+\ell_{\Omega \backslash \Lambda}(\delta U) .
$$

We then introduce a relaxation

$$
U_{n}=\rho \hat{U}_{n}+(1-\rho) U_{n-1},
$$

with $\rho>0$. Global step (10) is a problem defined on the domain $\widetilde{\Omega}$, with an arbitrary diffusion parameter $\widetilde{K}$ in $\widetilde{\Lambda}$ and which does not contain any geometrical details in $\widetilde{\Lambda}$. The term $\lambda_{n-1}$ appears as an imposed source term on an internal surface in $\widetilde{\Omega}$.

Local step. We then compute $\left(w_{n}, \lambda_{n}\right) \in \mathcal{W}^{\Xi} \times \mathcal{M}^{\Xi}$ such that for all $(\delta w, \delta \lambda) \in$ $\mathcal{W}^{\Xi} \times \mathcal{M}^{\Xi}$

$$
\left\{\begin{array}{rl}
a_{\Lambda}\left(w_{n}, \delta w\right)-b_{\Gamma}\left(\lambda_{n}, \delta w\right) & =\ell_{\Lambda}(\delta w) \\
b_{\Gamma}\left(\delta \lambda, w_{n}\right) & =b_{\Gamma}\left(\delta \lambda, U_{n}\right)
\end{array} .\right.
$$

Local step (11) is a problem defined on the patch $\Lambda$, with the true material parameter $K$ and the true geometrical details. $\lambda_{n}$ can be interpreted as a Lagrange multiplier that ensures in a weak sense $w_{n}=U_{n}$ on $\Gamma$.

Remark 1. Note that the local problem (11) can be reformulated by introducing a change of variable $w_{n}=U_{n}^{\diamond}+z_{n}$, with $U_{n}^{\diamond} \in \widetilde{\mathcal{W}}^{\Xi}$ satisfying $b\left(\delta \lambda, U_{n}^{\diamond}-U_{n}\right)=0$ for all $\delta \lambda \in \mathcal{M}^{\Xi}$ (i.e. $U_{n}^{\diamond}=U_{n}$ on $\Gamma$ in a weak sense), and with

$$
z_{n} \in \mathcal{W}_{0}^{\Xi}=\left\{z \in \mathcal{W}^{\Xi} ; z=0 \text { on } \Gamma\right\} .
$$

Local problem (11) can then be formulated as follows:

$$
a_{\Lambda}\left(z_{n}, \delta w\right)=\ell_{\Lambda}(\delta w)-a_{\Lambda}\left(U_{n}^{\diamond}, \delta w\right) \quad \forall \delta w \in \mathcal{W}_{0}^{\Xi} .
$$

The Lagrange multiplier $\lambda_{n}$ is then obtained from the first equation of (11). 


\subsection{Prolongation in the fictitious patch is uniquely defined}

The prolongation of $U_{n}$ in the fictitious patch is uniquely defined by the initialization $U_{0}$. Indeed, from $(10)$, we have that for all $\delta U \in H_{0}^{1}(\widetilde{\Lambda}) \otimes L_{P_{\xi}}^{2}(\Xi)$,

$$
c_{\widetilde{\Lambda}}\left(\hat{U}_{n}, \delta U\right)=c_{\widetilde{\Lambda}}\left(U_{n-1}, \delta U\right),
$$

and therefore

$$
c_{\widetilde{\Lambda}}\left(U_{n}, \delta U\right)=c_{\widetilde{\Lambda}}\left(\rho \hat{U}_{n}+(1-\rho) U_{n-1}, \delta U\right)=c_{\widetilde{\Lambda}}\left(U_{n-1}, \delta U\right) .
$$

We then have that for all $\delta U \in H_{0}^{1}(\widetilde{\Lambda}) \otimes L_{P_{\xi}}^{2}(\Xi)$,

$$
c_{\widetilde{\Lambda}}\left(U_{n}, \delta U\right)=c_{\widetilde{\Lambda}}\left(U_{0}, \delta U\right)
$$

for all $n \geq 1$. With $U_{0}=0$, we obtain that all iterates $U_{n} \in \widetilde{\mathcal{U}}^{\Xi}$ belongs to a subspace $\widetilde{\mathcal{U}}_{*}^{\Xi} \subset \widetilde{\mathcal{U}}^{\Xi}$ defined by

$$
\widetilde{\mathcal{U}}_{*}^{\Xi}=\left\{U \in \widetilde{\mathcal{U}}^{\Xi} ; c_{\widetilde{\Lambda}}(U, \delta U)=0 \text { for all } \delta U \in H_{0}^{1}(\widetilde{\Lambda}) \otimes L_{P_{\xi}}^{2}(\Xi)\right\} .
$$

$U_{n}$ can be interpreted as the unique analytical prolongation into $\widetilde{\mathcal{U}}^{\Xi}$ of $U_{n} \in \mathcal{U}^{\Xi}$. The restriction of $U_{n}$ on $\widetilde{\Lambda}$ is then uniquely defined by the trace of $U_{n}$ on $\Gamma$. Problem (9) appears to be well-posed in the subspace $\widetilde{\mathcal{U}}_{*}^{\Xi} \times \mathcal{W}^{\Xi} \times \mathcal{M}^{\Xi} \subset$ $\tilde{\mathcal{U}}^{\Xi} \times \mathcal{W}^{\Xi} \times \mathcal{M}^{\Xi}$, which gives a sense to the prolongation of the solution $U$. In the following, we use the following definition for $U$.

Definition 1. We denote by $U \in \widetilde{\mathcal{U}}_{*}^{\Xi} \subset \widetilde{\mathcal{U}}^{\Xi}$ the prolongation of the solution $U \in \mathcal{U}^{\Xi}$ of (4). This prolongation is such that $(U, \lambda, w) \in \tilde{\mathcal{U}}_{*}^{\Xi} \times \mathcal{W}^{\Xi} \times \mathcal{M}^{\Xi}$ is the unique solution of equation (9) for all $(\delta U, \delta w, \delta \lambda) \in \widetilde{\mathcal{U}}_{*}^{\Xi} \times \mathcal{W}^{\Xi} \times \mathcal{M}^{\Xi}$.

The convergence of the sequence $U_{n}$ to this solution $U \in \widetilde{\mathcal{U}}_{*}^{\Xi}$ is now analyzed.

\subsection{Convergence analysis}

Let introduce the mapping $\Phi: \mathcal{M}^{\Xi} \times \widetilde{\mathcal{U}}^{\Xi} \rightarrow \widetilde{\mathcal{U}}^{\Xi}$ such that $\Phi(\beta, V) \in \tilde{\mathcal{U}}^{\Xi}$ is solution of:

$$
c_{\widetilde{\Omega}}(\Phi(\beta, V), \delta U)=c_{\widetilde{\Lambda}}(V, \delta U)-b_{\Gamma}(\beta, \delta U) .
$$

Let us also introduce the mappings $\Psi: \widetilde{\mathcal{U}}^{\Xi} \rightarrow \mathcal{M}^{\Xi}$ and $\psi: \widetilde{\mathcal{U}}^{\Xi} \rightarrow \mathcal{W}^{\Xi}$ such that the couple $(\Psi(V), \psi(V)) \in \mathcal{M}^{\Xi} \times \mathcal{W}^{\Xi}$ is solution of:

$$
\left\{\begin{array}{rl}
a_{\Lambda}(\psi(V), \delta w)-b_{\Gamma}(\Psi(V), \delta w) & =0 \\
b_{\Gamma}(\delta \lambda, \psi(V)) & =b_{\Gamma}(\delta \lambda, V)
\end{array} \quad \forall(\delta w, \delta \lambda) \in \mathcal{W}^{\Xi} \times \mathcal{M}^{\Xi} .\right.
$$

Let $(U, \lambda, w) \in \widetilde{\mathcal{U}}_{*}^{\Xi} \times \mathcal{M}^{\Xi} \times \mathcal{W}^{\Xi}$ be the exact solution of (9). With the above definitions, the proposed algorithm is equivalent to:

$$
\begin{aligned}
& \hat{U}_{n}-U=\Phi\left(\lambda_{n-1}-\lambda, U_{n-1}-U\right), \\
& U_{n}-U=\rho\left(\hat{U}_{n}-U\right)+(1-\rho)\left(U_{n-1}-U\right), \\
& \left(\lambda_{n}-\lambda, w_{n}-w\right)=\left(\Psi\left(U_{n}-U\right), \psi\left(U_{n}-U\right)\right) .
\end{aligned}
$$


Letting $E_{n}=U_{n}-U$ the error at iteration $n$, we have

$$
E_{n}=\rho \Phi\left(\Psi\left(E_{n-1}\right), E_{n-1}\right)+(1-\rho) E_{n-1}:=B_{\rho}\left(E_{n-1}\right),
$$

with the iteration operator $B_{\rho}$ defined by

$$
B_{\rho}(V)=\rho \Phi(\Psi(V), V)+(1-\rho) V .
$$

Lemma 2. The iteration operator $B_{\rho}: \widetilde{\mathcal{U}}^{\Xi} \rightarrow \widetilde{\mathcal{U}}^{\Xi}$ defined in (17) is such that

$$
B_{\rho}=I-\rho A,
$$

with $A: \widetilde{\mathcal{U}}^{\Xi} \rightarrow \widetilde{\mathcal{U}}^{\Xi}$ such that $A(V) \in \tilde{\mathcal{U}}^{\Xi}$ is solution of

$$
c_{\widetilde{\Omega}}(A(V), \delta V)=b_{\Gamma}(\Psi(V), \delta V)+a_{\Omega \backslash \Lambda}(V, \delta V) \quad \forall \delta V \in \widetilde{\mathcal{U}}^{\Xi} .
$$

Proof. From definition (17) of $B_{\rho}$, it follows immediately

$$
A(V)=V-\Phi(\Psi(V), V)
$$

From (13), we have that $\Phi(\Psi(V), V) \in \widetilde{\mathcal{U}}^{\Xi}$ is defined by the following equation:

$$
c_{\widetilde{\Omega}}(\Phi(\Psi(V), V), \delta V)=-b_{\Gamma}(\Psi(V), \delta V)+c_{\widetilde{\Lambda}}(V, \delta V) \quad \forall \delta V \in \widetilde{\mathcal{U}}^{\Xi} .
$$

Using (8), it yields the desired expression (18).

Note that $U$ and the iterates $U_{n}$ belong to the subspace $\widetilde{\mathcal{U}}_{*}^{\Xi} \subset \widetilde{\mathcal{U}}^{\Xi}$, so that only the restriction to $\mathcal{U}_{*}^{\Xi}$ of operator $A$ defined in (18) has to be analyzed.

Lemma 3. The operator $A: \widetilde{\mathcal{U}}_{*}^{\Xi} \rightarrow \widetilde{\mathcal{U}}_{*}^{\Xi}$ is symmetric, bounded and coercive.

Proof. Since $\widetilde{K}$ is uniformly bounded and elliptic, we can equip the Hilbert space $\widetilde{\mathcal{U}}_{*}^{\Xi} \subset \widetilde{\mathcal{U}}^{\Xi}$ with the inner product

$$
\langle U, V\rangle_{\widetilde{\Omega}, \widetilde{K}}=c_{\widetilde{\Omega}}(U, V)=\mathbb{E}\left(\int_{\widetilde{\Omega}} \widetilde{K} \nabla U \cdot \nabla V\right)
$$

and the associated norm $\|V\|_{\widetilde{\Omega}, \widetilde{K}}=\sqrt{c_{\widetilde{\Omega}}(V, V)}$. From properties of $\widetilde{K},\|\cdot\|_{\widetilde{\Omega}, \widetilde{K}}$ is equivalent to $\|\cdot\|_{\widetilde{\Omega}}$. From (18), operator $A$ is such that

$$
\langle A(U), V\rangle_{\widetilde{\Omega}, \widetilde{K}}=c_{\widetilde{\Omega}}(A(U), V)=b_{\Gamma}(\Psi(U), V)+a_{\Omega \backslash \Lambda}(U, V) .
$$

Using for test functions $(\delta \lambda, \delta w)=(\Psi(U), \psi(U))$ in equation (14), we obtain

$$
\left\{\begin{array}{l}
a_{\Lambda}(\psi(V), \psi(U))=b_{\Gamma}(\Psi(V), \psi(U)) \\
b_{\Gamma}(\Psi(U), \psi(V))=b_{\Gamma}(\Psi(U), V)
\end{array}\right.
$$

Equations (20) are also verified if we permute $U$ and $V$. We then obtain

$$
b_{\Gamma}(\Psi(U), V)=b_{\Gamma}(\Psi(U), \psi(V))=a_{\Lambda}(\psi(U), \psi(V))=b_{\Gamma}(\Psi(V), U),
$$


where we have used the symmetry of $a_{\Lambda}$. That proves that operator $A$ is symmetric. The continuity of $A$ follows directly from its definition as the solution of the variational problem (18) involving a symmetric continuous and coercive bilinear form. Moreover,

$$
b_{\Gamma}(\Psi(U), U)=a_{\Lambda}(\psi(U), \psi(U)) \geq 0,
$$

and therefore

$$
\langle A(U), U\rangle_{\widetilde{\Omega}, \widetilde{K}} \geq a_{\Omega \backslash \Lambda}(U, U) \gtrsim\|U\|_{\Omega \backslash \Lambda}^{2},
$$

where the last inequality is obtained from the property of coercivity of $a_{\Omega \backslash \Lambda}$ on $\mathcal{U}^{\Xi}$. Finally, it remains to see that on $\widetilde{\mathcal{U}}_{*}^{\Xi},\|\cdot\|_{\Omega \backslash \Lambda}$ defines a norm which is equivalent to the norm $\|\cdot\|_{\tilde{\Omega}}$, and therefore:

$$
\langle A(U), U\rangle_{\widetilde{\Omega}, \widetilde{K}} \gtrsim\|U\|_{\widetilde{\Omega}}^{2} \gtrsim\|U\|_{\widetilde{\Omega}, \widetilde{K}}^{2}
$$

which proves that $A$ is coercive on the subspace $\widetilde{\mathcal{U}}_{*}^{\Xi}$ of $\widetilde{\mathcal{U}}^{\Xi}$.

We finally state the following convergence result.

Theorem 4. The sequence $U_{n}$ converges towards $U$ for a sufficiently small relaxation parameter $\rho>0$.

Proof. Since $A$ is symmetric, bounded and coercive, we have

$$
\begin{aligned}
\left\|B_{\rho}(U)-B_{\rho}(V)\right\|^{2} & =\|U-V-\rho(A(U)-A(V))\|^{2} \\
& \leq\left(1-2 \rho \alpha_{A}+\rho^{2} C_{A}^{2}\right)\|U-V\|^{2}
\end{aligned}
$$

with $\alpha_{A}$ and $C_{A}$ the coercivity and continuity constants of $A$ respectively. Choosing $0<\rho<\frac{2 \alpha_{A}}{C_{A}^{2}}$, we have $\rho_{B}=\left(1-2 \rho \alpha_{A}+\rho^{2} C_{A}^{2}\right)^{1 / 2}<1$ and therefore, $B_{\rho}$ is a contractive mapping with contractivity constant $\rho_{B}$. The error $\varepsilon_{n}=\left\|U_{n}-U\right\|$ then verifies

$$
\varepsilon_{n}=\left\|B_{\rho}\left(U_{n-1}\right)-B_{\rho}(U)\right\| \leq \rho_{B} \varepsilon_{n-1} \leq \rho_{B}^{n} \varepsilon_{0},
$$

which yields $\varepsilon_{n} \rightarrow 0$ as $n \rightarrow \infty$.

Remark 2. Note that if $\widetilde{K}=K$ on $\widetilde{\Lambda}$ and if $\Lambda=\widetilde{\Lambda}$, we have $A=I$, so that $B_{\rho}=(1-\rho) I$, yielding to convergence for $0<\rho<2$. This is also true in the discretized framework when using Galerkin projections and when the approximation space in $\mathcal{W}^{\Xi}$ is the restriction to $\widetilde{\Lambda}$ of functions of the approximation space in $\widetilde{\mathcal{U}}^{\Xi}$.

Remark 3. Note that the final solution does not depend on the choice of $\widetilde{K}$. However, this choice influences the behavior of the iterative algorithm (it affects the spectrum of operator A), as it will be illustrated in numerical examples. A convenient choice consists in taking for $\widetilde{K}$ a deterministic field, yielding to a 
global problem with deterministic operator. A natural choice yielding rather good convergence properties consists in taking for $\widetilde{K}$ the mean diffusion field:

$$
\widetilde{K}(x)=\mathbb{E}(K(x, \xi)) .
$$

However, we will see in numerical examples that a large range of values for the fictitious field $\widetilde{K}$ yield good convergence properties, thus providing flexibility in the definition of the global problem.

\subsection{Robustness of the algorithm with respect to approximations}

We suppose that approximations are introduced in the different solution steps, thus defining an approximate sequence $U_{n}^{\epsilon} \in \widetilde{\mathcal{U}}_{*}^{\Xi}$ such that

$$
U_{n}^{\epsilon}-U=B_{\rho}^{\epsilon}\left(U_{n-1}^{\epsilon}-U\right),
$$

where $B_{\rho}^{\epsilon}$ is a reasonable approximation of the iteration operator $B_{\rho}$. We assume that

$$
B_{\rho}^{\epsilon}\left(U_{n-1}^{\epsilon}-U\right)=B_{\rho}\left(U_{n-1}^{\epsilon}-U\right)+\delta U_{n}^{\epsilon}
$$

where the perturbation $\delta U_{n}^{\epsilon}$ verifies

$$
\left\|\delta U_{n}^{\epsilon}\right\| \leq \epsilon\|U\|+\epsilon^{*}\left\|U_{n-1}^{\epsilon}-U\right\| .
$$

$\epsilon$ is associated with a tolerance relatively to the solution norm, and $\epsilon^{*}$ is associated with a tolerance relatively to the current error. $\epsilon^{*}$ typically appears when formulating local and global problems on increments $\left(U_{n}^{\epsilon}-U_{n-1}^{\epsilon}\right)$ and $\left(\lambda_{n}^{\epsilon}-\lambda_{n-1}^{\epsilon}\right)$ and when using solvers with a prescribed tolerance of the order of $\epsilon^{*}$. $\epsilon$ typically appears when local and global problems are not formulated on increments and when we use solvers with a prescribed tolerance of the order of $\epsilon$. This error $\epsilon$ can also appear when we introduce some additional approximation of the iterates, typically when introducing spectral decompositions in order to "compress" the representations of the iterates (see section 7). We now provide a result that gives the behavior of the algorithm with respect to these two types of errors.

Theorem 5. We suppose that

$$
\begin{aligned}
& \left\|B_{\rho}^{\epsilon}(V)-B_{\rho}(V)\right\| \leq \epsilon\|U\|+\epsilon^{*}\|V\| \\
& \text { for all } V \in \widetilde{\mathcal{U}}_{*}^{\Xi} \text { such that }\|V\| \leq \eta\|U\|,
\end{aligned}
$$

with $\eta>0, \epsilon^{*}<1-\rho_{B}$ and $\epsilon \leq \eta\left(1-\rho_{B}-\epsilon^{*}\right)$. We assume that $\left\|U_{0}^{\epsilon}-U\right\| \leq \eta\|U\|$. Then, the sequence $U_{n}^{\epsilon}$ is such that

$$
\limsup _{n \rightarrow \infty}\left\|U_{n}^{\epsilon}-U\right\| \leq \gamma(\epsilon)\|U\|,
$$

with $\gamma(\epsilon) \rightarrow 0$ as $\epsilon \rightarrow 0$. That means that the sequence $U_{n}^{\epsilon}$ tends to a $\gamma(\epsilon)$ neighborhood of the solution $U$. 
Proof. We denote $E_{n}^{\epsilon}=U_{n}^{\epsilon}-U$. We have $\left\|E_{0}^{\epsilon}\right\| \leq \eta\|U\|$. Let us suppose that $\left\|E_{m}^{\epsilon}\right\| \leq \eta\|U\|$ for all $m<n$. Then,

$$
\begin{aligned}
\left\|E_{n}^{\epsilon}\right\| & =\left\|B_{\rho}^{\epsilon}\left(E_{n-1}^{\epsilon}\right)\right\| \\
& \leq\left\|B_{\rho}^{\epsilon}\left(E_{n-1}^{\epsilon}\right)-B_{\rho}\left(E_{n-1}^{\epsilon}\right)\right\|+\left\|B_{\rho}\left(E_{n-1}^{\epsilon}\right)\right\| \\
& \leq \epsilon\|U\|+\left(\rho_{B}+\epsilon^{*}\right)\left\|E_{n-1}^{\epsilon}\right\| .
\end{aligned}
$$

Since $\epsilon \leq \eta\left(1-\rho_{B}-\epsilon^{*}\right)$, we then obtain $\left\|E_{n}^{\epsilon}\right\| \leq \eta\|U\|$ for all $n$. Finally, we obtain

$$
\begin{aligned}
\left\|E_{n}^{\epsilon}\right\| & \leq \frac{\epsilon\left(1-\left(\rho_{B}+\epsilon^{*}\right)^{n}\right)}{1-\left(\rho_{B}+\epsilon^{*}\right)}\|U\|+\left(\rho_{B}+\epsilon^{*}\right)^{n}\left\|E_{0}^{\epsilon}\right\| \\
& \leq \frac{\epsilon}{1-\rho_{B}-\epsilon^{*}}\|U\|+\left(\rho_{B}+\epsilon^{*}\right)^{n}\left\|E_{0}^{\epsilon}\right\|,
\end{aligned}
$$

and therefore

$$
\limsup _{n \rightarrow \infty}\left\|E_{n}^{\epsilon}\right\| \leq \frac{\epsilon}{1-\rho_{B}-\epsilon^{*}}\|U\|:=\gamma(\epsilon)\|U\|,
$$

with $\gamma(\epsilon)=\frac{\epsilon}{1-\rho_{B}-\epsilon^{*}} \rightarrow 0$ as $\epsilon \rightarrow 0$.

Remark 4. Note that when $\epsilon$ is of the order of the machine precision, then the sequence $U_{n}^{\epsilon}$ converges to the solution $U$ down to the machine precision times a factor $\left(1-\rho_{B}-\epsilon^{*}\right)^{-1}$. It is the case when we solve local and global problems formulated on increments with a tolerance $\epsilon^{*}$, and when additional errors are of the order of the machine precision. The sequence $U_{n}^{\epsilon}$ also converges to the solution $U$ down to the machine precision when solving local problems formulated on increments with prescribed tolerance $\epsilon^{*}$, and when solving global problems at the machine precision, which is feasible if the global problem only involves a deterministic operator.

\section{Approximate solutions of local and global problems}

\subsection{Approximation spaces}

We introduce finite element meshes $\mathcal{T}_{H}^{\widetilde{\Omega}}$ and $\mathcal{T}_{h}^{\Lambda}$ of $\widetilde{\Omega}$ and $\Lambda$ respectively. We suppose that $\mathcal{T}_{H}^{\widetilde{\Omega}}=\mathcal{T}_{H}^{\Omega \backslash \Lambda} \cup \mathcal{T}_{H}^{\widetilde{\Lambda}}$, with $\mathcal{T}_{H}^{\Omega \backslash \Lambda}$ a mesh of $\Omega \backslash \Lambda$ and $\mathcal{T}_{H}^{\widetilde{\Lambda}}$ a mesh of $\widetilde{\Lambda}$, such that the interface $\Gamma$ coincides with the intersection between the boundaries of meshes $\mathcal{T}_{H}^{\Omega \backslash \Lambda}$ and $\mathcal{T}_{H}^{\widetilde{\Lambda}}$. We also suppose that the interface $\Gamma$ is contained in the boundary of the mesh $\mathcal{T}_{h}^{\Lambda}$. We introduce finite element approximation spaces $\tilde{\mathcal{U}}_{H} \subset \widetilde{\mathcal{U}}, \mathcal{W}_{h} \subset \mathcal{W}$ and $\mathcal{M}_{h} \subset \mathcal{M}$. In this paper, we consider meshes $\mathcal{T}_{H}^{\widetilde{\Omega}}$ and $\mathcal{T}_{h}^{\Lambda}$ that are eventually not conforming on $\Gamma$. The approximation space $\mathcal{M}_{h}$ for the Lagrange multiplier will be simply chosen as the trace on $\Gamma$ of the approximation space $\mathcal{W}_{h}$. For the construction of suitable approximation spaces $\mathcal{M}_{h}$ in the case of non conforming meshes, see [31] and the references therein. 
A finite element approximation space $\mathcal{H}$ with dimension $N$ and basis $\left\{\varphi_{i}\right\}_{i=1}^{N}$ being given, an element $v \in \mathcal{H}$ will be associated with a vector $\mathbf{v}=\left(v_{i}\right)_{i=1}^{N} \in \mathbb{R}^{N}$ such that $v=\sum_{i=1}^{N} v_{i} \varphi_{i}$. An element $v \in \mathcal{H}^{\Xi}$ will be associated with a random vector $\mathbf{v}=\left(v_{i}\right)_{i=1}^{N} \in\left(\mathbb{R}^{N}\right)^{\Xi}$, such that $v(\xi)=\sum_{i=1}^{N} v_{i}(\xi) \varphi_{i}$. We then introduce finite element matrices and vectors such that

$$
\begin{aligned}
& c_{\mathcal{O}}(U, V)=\mathbb{E}\left(\mathbf{V}^{T} \mathbf{C}_{\mathcal{O}} \mathbf{U}\right), \quad a_{\mathcal{O}}(U, V)=\mathbb{E}\left(\mathbf{V}^{T} \mathbf{A}_{\mathcal{O}} \mathbf{U}\right), \\
& \ell_{\mathcal{O}}(V)=\mathbb{E}\left(\mathbf{V}^{T} \mathbf{L}_{\mathcal{O}}\right) .
\end{aligned}
$$

For the interface coupling bilinear form $b_{\Gamma}(\cdot, \cdot)$, we distinguish the bilinear form when defined on $\mathcal{M}_{h}^{\Xi} \times \widetilde{\mathcal{U}}_{H}^{\Xi}$ or on $\mathcal{M}_{h}^{\Xi} \times \mathcal{W}_{h}^{\Xi}$ :

$$
\begin{array}{ll}
b_{\Gamma}(\lambda, V)=\mathbb{E}\left(\mathbf{V}^{T} \widetilde{\mathbf{B}}_{\Gamma} \boldsymbol{\lambda}\right) & \text { for } V \in \widetilde{\mathcal{U}}_{H}^{\Xi}, \\
b_{\Gamma}(\lambda, w)=\mathbb{E}\left(\mathbf{w}^{T} \mathbf{B}_{\Gamma} \boldsymbol{\lambda}\right) & \text { for } w \in \mathcal{W}_{h}^{\Xi} .
\end{array}
$$

We also introduce an approximation space (e.g. polynomial space, finite element space)

$$
\mathcal{S}_{P}=\left\{v(\xi)=\sum_{k=1}^{P} v_{k} \Psi_{k}(\xi)\right\} \subset \mathcal{S} .
$$

An element $v \in \mathcal{H} \otimes \mathcal{S}_{P}$ can then be written $v=\sum_{i=1}^{N} \sum_{k=1}^{P} v_{k, i} \varphi_{i} \otimes \Psi_{k}$, and identified with an element $\mathbf{v} \in \mathbb{R}^{N} \otimes \mathcal{S}_{P}$ such that $\mathbf{v}=\sum_{k=1}^{P} \mathbf{v}_{k} \otimes \Psi_{k}$, with $\mathbf{v}_{k}=\left(v_{k, i}\right)_{i=1}^{N} \in \mathbb{R}^{N}$.

\subsection{Global problem}

The approximate global problem consists in finding $\hat{U}_{n} \in \widetilde{\mathcal{U}}_{H} \otimes \mathcal{S}_{P}$ such that

$$
c_{\widetilde{\Omega}}\left(\hat{U}_{n}, \delta U\right)=c_{\widetilde{\Lambda}}\left(U_{n-1}, \delta U\right)-b_{\Gamma}\left(\lambda_{n-1}, \delta U\right)+\ell_{\Omega \backslash \Lambda}(\delta U) \quad \forall \delta U \in \widetilde{\mathcal{U}}_{H} \otimes \mathcal{S}_{P} .
$$

It reduces to the system of algebraic equations

$$
\mathbb{E}\left(\delta \mathbf{U}^{T} \mathbf{C}_{\widetilde{\Omega}} \hat{\mathbf{U}}_{n}\right)=\mathbb{E}\left(\delta \mathbf{U}^{T} \mathbf{d}\right) \quad \forall \delta \mathbf{U} \in \mathbb{R}^{N_{U}} \otimes \mathcal{S}_{P}
$$

with

$$
\mathbf{d}=\mathbf{C}_{\widetilde{\Lambda}} \mathbf{U}_{n-1}-\widetilde{\mathbf{B}}_{\Gamma} \boldsymbol{\lambda}_{n-1}+\mathbf{L}_{\Omega \backslash \Lambda} .
$$

If the fictitious diffusion parameter $\widetilde{K}$ does not depend on the uncertain parameters $\xi$, finite element matrix $\mathbf{C}_{\widetilde{\Omega}} \in \mathbb{R}^{N_{U} \times N_{U}}$ also does not depend on the uncertain parameters. If $\mathbf{d} \in \mathbb{R}^{N_{U}} \otimes \mathcal{S}_{P}$ is given under the form

$$
\mathbf{d}=\sum_{i=1}^{m} \mathbf{d}_{i} \otimes \gamma_{i}
$$

with $\mathbf{d}_{i} \in \mathbb{R}^{N_{U}}$ and $\gamma_{i} \in \mathcal{S}_{P}$, then we directly obtain an exact decomposition of the solution $\hat{\mathbf{U}}_{n}$ under the form

$$
\hat{\mathbf{U}}_{n}=\sum_{i=1}^{m}\left(\mathbf{C}_{\widetilde{\Omega}}^{-1} \mathbf{d}_{i}\right) \otimes \gamma_{i} .
$$




\subsection{Local problems with uncertain operator and right-hand side}

The approximate local problem consists in finding $\left(w_{n}, \lambda_{n}\right) \in\left(\mathcal{W}_{h} \otimes \mathcal{S}_{P}\right) \times$ $\left(\mathcal{M}_{h} \otimes \mathcal{S}_{P}\right)$ such that

$$
\left\{\begin{aligned}
a_{\Lambda}\left(w_{n}, \delta w\right)-b_{\Gamma}\left(\lambda_{n}, \delta w\right) & =\ell_{\Lambda}(\delta w) & & \forall \delta w \in \mathcal{W}_{h} \otimes \mathcal{S}_{P} \\
b_{\Gamma}\left(\delta \lambda, w_{n}\right) & =b_{\Gamma}\left(\delta \lambda, U_{n}\right) & & \forall \delta \lambda \in \mathcal{M}_{h} \otimes \mathcal{S}_{P}
\end{aligned}\right.
$$

It reduces to the following system of algebraic equations

$$
\begin{cases}\mathbb{E}\left(\delta \mathbf{w}^{T} \mathbf{A}_{\Lambda} \mathbf{w}_{n}\right)-\mathbb{E}\left(\delta \mathbf{w}^{T} \mathbf{B}_{\Gamma} \boldsymbol{\lambda}_{n}\right)=\mathbb{E}\left(\delta \mathbf{w}^{T} \mathbf{L}_{\Lambda}\right) & \forall \delta \mathbf{w} \in \mathbb{R}^{N_{w}} \otimes \mathcal{S}_{P} \\ \mathbb{E}\left(\delta \boldsymbol{\lambda}^{T} \mathbf{B}_{\Gamma}^{T} \mathbf{w}_{n}\right)=\mathbb{E}\left(\delta \boldsymbol{\lambda}^{T} \widetilde{\mathbf{B}}_{\Gamma} \mathbf{U}_{n}\right) & \forall \delta \boldsymbol{\lambda} \in \mathbb{R}^{N_{\lambda}} \otimes \mathcal{S}_{P}\end{cases}
$$

Following Remark 1, we note that problem (26) can be reformulated by introducing a change of variable $w_{n}=U_{n}^{\diamond}+z_{n}$, with $U_{n}^{\diamond} \in \mathcal{W}_{h} \otimes \mathcal{S}_{P}$ a prolongation of $U_{n}$ from $\Gamma$ to $\Lambda$ such that

$$
b_{\Gamma}\left(\delta \lambda, U_{n}^{\diamond}-U_{n}\right)=0 \quad \forall \delta \lambda \in \mathcal{M}_{h} \otimes \mathcal{S}_{P},
$$

and $z_{n} \in \mathcal{W}_{h, 0} \otimes \mathcal{S}_{P}$, with $\mathcal{W}_{h, 0}=\left\{z \in \mathcal{W}_{h} ; z=0\right.$ on $\left.\Gamma\right\} . z_{n}$ is then solution of

$$
a_{\Lambda}\left(z_{n}, \delta z\right)=\ell_{\Lambda}(\delta z)-a_{\Lambda}\left(U_{n}^{\diamond}, \delta z\right) \quad \forall \delta z \in \mathcal{W}_{h, 0} \otimes \mathcal{S}_{P}
$$

$\lambda_{n}$ is then obtained in a post-processing step using the first equation of (26).

\subsection{Local problems with geometrical variabilities}

The solution method presented in section 4.3 is valid when the patch contains geometrical details with a fixed internal boundary $\Gamma^{\Lambda}:=\partial \Lambda \backslash \Gamma$. It suffices to introduce a conforming approximation space $\mathcal{W}_{h}$ in $\mathcal{W}$. However, if the patch $\Lambda$ contains uncertain geometrical details, that means an uncertain internal boundary $\Gamma^{\Lambda}(\xi)$, the previous construction of approximation spaces is not possible since the space $\mathcal{W}^{\Xi}$ has no more a tensor product structure. Different strategies have been proposed in the literature for solving such problems. They are based on reformulation of the problem on a deterministic domain, by introducing a suitable random mapping to a fixed deterministic domain [32], or by introducing reformulations on a deterministic fictitious domain $[28,29,30]$. We here present two types of reformulations based on the introduction of a fictitious domain, the different reformulations depending on the type of boundary conditions (Dirichlet or Neumann) that are imposed on the internal boundary $\Gamma^{\Lambda}$.

\subsubsection{Fictitious domain and approximation spaces}

In the present context, a natural choice consists in prolongating function $w$ on the fictitious patch $\widetilde{\Lambda}$. We then consider functions $w$ in $\mathcal{W}^{\Xi}$, with $\widetilde{\mathcal{W}}=$

$H^{1}(\widetilde{\Lambda})$. Function space $\widetilde{\mathcal{W}}^{\Xi}$ can now be identified with the tensor product space $\widetilde{\mathcal{W}} \otimes \mathcal{S}$ and approximation spaces can be introduced such as in section 4.1, i.e. 
by introducing a finite element approximation space $\widetilde{\mathcal{W}}_{h} \subset \widetilde{\mathcal{W}}$ (associated with a mesh $\mathcal{T}_{h}^{\widetilde{\Lambda}}$ ) and the approximation space $\mathcal{S}_{P} \subset \mathcal{S}$. We also introduce the function space $\widetilde{\mathcal{W}}_{0}=\{v \in \widetilde{\mathcal{W}} ; v=0$ on $\Gamma\}$ and the corresponding finite element space $\widetilde{\mathcal{W}}_{h, 0} \subset \widetilde{\mathcal{W}}_{0}$.

\subsubsection{Neumann internal boundary conditions}

We consider that $\Gamma^{\Lambda}$ is a Neumann boundary with homogeneous data. The local problem can then be reformulated on the fictitious domain as follows: find $\left(w_{n}, \lambda_{n}\right) \in\left(\widetilde{\mathcal{W}}_{h} \otimes \mathcal{S}_{P}\right) \times\left(\mathcal{M}_{h} \otimes \mathcal{S}_{P}\right)$ such that

$$
\left\{\begin{aligned}
d_{\widetilde{\Lambda}}\left(w_{n}, \delta w\right)-b_{\Gamma}\left(\lambda_{n}, \delta w\right) & =h_{\widetilde{\Lambda}}(\delta w) & & \forall \delta w \in \widetilde{\mathcal{W}}_{h} \otimes \mathcal{S}_{P} \\
b_{\Gamma}\left(\delta \lambda, w_{n}\right) & =b_{\Gamma}\left(\delta \lambda, U_{n}\right) & & \forall \delta \lambda \in \mathcal{M}_{h} \otimes \mathcal{S}_{P}
\end{aligned}\right.
$$

where bilinear form $d_{\widetilde{\Lambda}}$ and linear form $h_{\widetilde{\Lambda}}$ are defined by

$$
\begin{aligned}
& d_{\widetilde{\Lambda}}(w, \delta w)=\mathbb{E}\left(\int_{\widetilde{\Lambda}} K I_{\Lambda} \nabla w \cdot \nabla \delta w\right), \\
& h_{\widetilde{\Lambda}}(\delta w)=\mathbb{E}\left(\int_{\widetilde{\Lambda}} I_{\Lambda} f \delta w\right) .
\end{aligned}
$$

with $I_{\Lambda}: \widetilde{\Lambda} \times \Xi \rightarrow \mathbb{R}$ the indicator function of the domain $\Lambda * \Xi=\{(x, y) \in$ $\widetilde{\Lambda} \times \Xi ; x \in \Lambda(y)\}$, such that $I_{\Lambda}(x, y)=1$ if $x \in \Lambda(y)$ and $I_{\Lambda}(x, y)=0$ if $x \notin \Lambda(y)$.

Again, with a suitable change of variable $w_{n}=U_{n}^{\diamond}+z_{n}$ (see remark 1), we can reformulate the local problem on $z_{n} \in \widetilde{\mathcal{W}}_{h, 0} \otimes \mathcal{S}_{P}$ such that

$$
d_{\widetilde{\Lambda}}\left(z_{n}, \delta w\right)=h_{\widetilde{\Lambda}}(\delta w)-d_{\widetilde{\Lambda}}\left(U_{n}^{\diamond}, \delta w\right) \quad \forall \delta w \in \widetilde{\mathcal{W}}_{h, 0} \otimes \mathcal{S}_{P}
$$

For further details on the numerical solution of such problems, see $[29,30]$.

\subsubsection{Dirichlet internal boundary conditions}

We now consider the case where $\Gamma^{\Lambda}$ is a Dirichlet boundary (with homogeneous data). We here propose a reformulation based on the use of a characteristic function method [33]. We introduce a characteristic function $\psi: \widetilde{\Lambda} \times \Xi \rightarrow \mathbb{R}$ such that

(i) $\psi(x, \xi)>0$ for $x \in \Lambda(\xi)$,

(ii) $\psi(x, \xi)=0$ for $x \in \Gamma_{\Lambda}(\xi)$,

(iii) $|\nabla \psi(x, \xi)| \neq 0$ for $x \in \Gamma_{\Lambda}(\xi)$.

A function $(\psi z)$ with $z \in \widetilde{\mathcal{W}}_{h} \otimes \mathcal{S}_{P}$ satisfies the Dirichlet boundary conditions on $\Gamma^{\Lambda}$. We then introduce a change of variable $w_{n}=U_{n}^{\diamond}+\psi z_{n}$, with $U_{n}^{\diamond}=U_{n}$ in a weak sense on $\Gamma$, and with $z_{n}=0$ on $\Gamma$. We then look for an approximation of the local problem under the form

$$
w_{n}=U_{n}^{\diamond}+\psi z_{n}
$$


with $z_{n} \in \widetilde{\mathcal{W}}_{h, 0} \otimes \mathcal{S}_{P}$ such that

$$
d_{\widetilde{\Lambda}}\left(\psi z_{n}, \psi \delta z\right)=h_{\widetilde{\Lambda}}(\psi \delta z)-d_{\widetilde{\Lambda}}\left(U_{n}^{\diamond}, \psi \delta z\right) \quad \forall \delta z \in \widetilde{\mathcal{W}}_{h, 0} \otimes \mathcal{S}_{P},
$$

with $d_{\widetilde{\Lambda}}$ and $h_{\widetilde{\Lambda}}$ prolongations of $a_{\Lambda}$ and $\ell_{\Lambda}$ on the fictitious patch:

$$
d_{\widetilde{\Lambda}}(w, \delta w)=\mathbb{E}\left(\int_{\widetilde{\Lambda}} K^{\diamond} \nabla w \cdot \nabla \delta w\right), \quad h_{\widetilde{\Lambda}}(\delta w)=\mathbb{E}\left(\int_{\widetilde{\Lambda}} f^{\diamond} \delta w\right),
$$

with $K^{\diamond}$ and $f^{\diamond}$ prolongations on $\widetilde{\Lambda}$ of $K$ and $f$ respectively.

\section{Multiple patches with independent variabilities}

We now consider the introduction of several patches containing uncertainties that are (statistically) independent between patches.

\subsection{Introduction of multiple patches}

The patch $\Lambda$ can be considered as the disjoint union of $N_{\Lambda}$ patches $\left\{\Lambda_{s}\right\}_{s=1}^{N_{\Lambda}}$. We suppose that the set of parameters can be split into disjoint subsets of parameters $\xi_{s}$ with values in $\Xi^{s} \subset \mathbb{R}^{d_{s}}\left(s=1, \ldots, N_{\Lambda}\right)$, such that $\Lambda_{s}$ only depends on $\xi_{s}$, i.e.

$$
\Lambda(\xi)=\Lambda_{1}\left(\xi_{1}\right) \cup \ldots \cup \Lambda_{N_{\Lambda}}\left(\xi_{N_{\Lambda}}\right),
$$

and the random field $K$ and source term $f$ also only depend on $\xi_{s}$ on the subdomain $\Lambda_{s}$, i.e.

$$
\begin{aligned}
& K(x, \xi)=K_{s}\left(x, \xi_{s}\right) \quad \text { for } \quad x \in \Lambda_{s}\left(\xi_{s}\right), \\
& f(x, \xi)=f_{s}\left(x, \xi_{s}\right) \quad \text { for } \quad x \in \Lambda_{s}\left(\xi_{s}\right),
\end{aligned}
$$

for all $s \in\left\{1, \ldots, N_{\Lambda}\right\}$.

\subsection{Local problems}

We denote $\Gamma_{s}=\Gamma \cap \partial \Lambda_{s}$ and $\Gamma_{D}^{\Lambda_{s}}=\Gamma_{D} \cap \partial \Lambda_{s}$. We then introduce

$$
\begin{aligned}
& \mathcal{W}_{s}=\left\{w \in H^{1}\left(\Lambda_{s}\right) ; w=0 \text { on } \Gamma_{D}^{\Lambda_{s}}\right\}, \\
& \mathcal{M}_{s}=H^{-1 / 2}\left(\Gamma_{s}\right),
\end{aligned}
$$

and the corresponding Bochner spaces $\mathcal{W}_{s}^{\Xi}$ and $\mathcal{M}_{s}^{\Xi}$. We have

$$
\mathcal{M}^{\Xi}=\times_{s=1}^{N_{\Lambda}} \mathcal{M}_{s}^{\Xi}, \quad \mathcal{W}^{\Xi}=\times_{s=1}^{N_{\Lambda}} \mathcal{W}_{s}^{\Xi},
$$

such that a function $\lambda \in \mathcal{M}^{\Xi}$ (resp. $w \in \mathcal{W}^{\Xi}$ ) is considered as a collection of local solutions $\lambda^{s} \in \mathcal{M}_{s}^{\Xi}$ (resp. $w^{s} \in \mathcal{W}_{s}^{\Xi}$ ). The local step is composed of $N_{\Lambda}$ independent problems on the $\Lambda_{s}$, that can be solved in parallel. At iteration $n$, denoting $w_{n}=\left\{w_{n}^{s}\right\}_{s=1}^{N_{\Lambda}}$ and $\lambda_{n}=\left\{\lambda_{n}^{s}\right\}_{s=1}^{N_{\Lambda}}$, we have for all $s \in\left\{1 \ldots N_{\Lambda}\right\}$,

$$
\left\{\begin{array}{rl}
a_{\Lambda_{s}}\left(w_{n}^{s}, \delta w\right)-b_{\Gamma_{s}}\left(\lambda_{n}^{s}, \delta w\right) & =\ell_{\Lambda_{s}}(\delta w) \\
b_{\Gamma_{s}}\left(\delta \lambda, w_{n}^{s}\right) & =b_{\Gamma_{s}}\left(\delta \lambda, U_{n}\right)
\end{array} \quad \forall(\delta \lambda, \delta w) \in \mathcal{M}_{s}^{\Xi} \times \mathcal{W}_{s}^{\Xi} .\right.
$$

For patches that contain geometrical details, the approximation methods presented in section 4.4 can be applied. 


\section{A first numerical example}

\subsection{Description of the example and approximation spaces}

We consider diffusion problem (1) on $\Omega=(0,5) \times(0,5)$ with $f=1, \Gamma_{D}=\partial \Omega$ and $\Gamma_{N}=\emptyset$. We define $\Lambda=\cup_{s=1}^{4} \Lambda_{s}$, where the four patches $\Lambda_{s}$ are square domains with side length $L=1$ and with respective centers $c_{1}=(3.5,3.5)$, $c_{2}=(1.5,1), c_{3}=(1.5,2.5)$, and $c_{4}=(3.5,1)$ (see Figure 3 ). We consider the diffusion field

$$
K(x, \xi)= \begin{cases}K_{0}=1 & \text { for } x \in \Omega \backslash \Lambda \\ K_{s}\left(x, \xi_{s}\right)=1+\xi_{s} k_{s}(x) & \text { for } x \in \Lambda_{s}, s \in\{1, \ldots, 4\}\end{cases}
$$

with $k_{s}(x)=\alpha \exp \left(-2 \frac{\left|x-c_{s}\right|^{2}}{0.25^{2}}\right)$ if $\max \left(x-c_{s}\right)<0.25$ and 0 if $\max \left(x-c_{s}\right) \geq$ 0.25 , and where $\xi=\left(\xi_{1}, \ldots, \xi_{4}\right)$ is a set of 4 independent uniform random variables with values in $\Xi=\times_{s=1}^{4} \Xi_{s}=(0,1)^{4}$. Constant $\alpha \in \mathbb{R}$ controls the level of heterogeneity. Here we have $\widetilde{\Omega}=\Omega$ and $\widetilde{\Lambda}=\Lambda$.

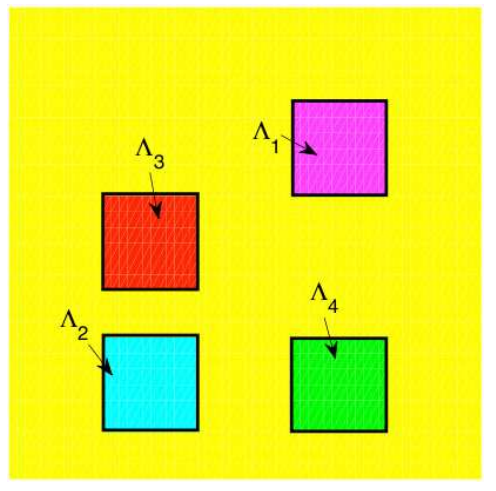

(a)

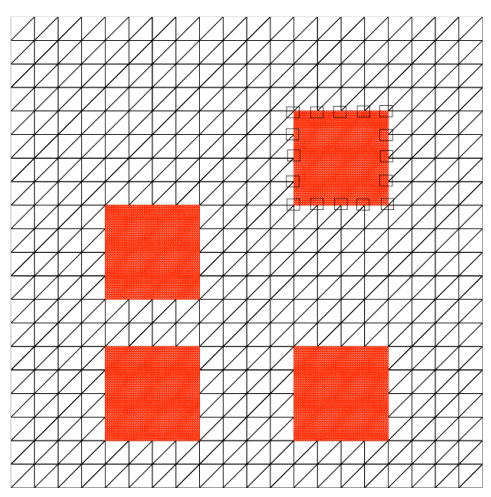

(b)

Figure 3: Example with localized heterogeneities. (a) Domain $\Omega$ and patches $\Lambda_{1}=(3,4) \times$ $(3,4), \Lambda_{2}=(1,2) \times(0.5,1.5), \Lambda_{3}=(1,2) \times(2,3), \Lambda_{4}=(3,4) \times(0.5,1.5)$. (b) Nested finite element meshes (the symbols $\square$ indicate the common nodes of $\mathcal{T}_{H}^{\Omega}$ and $\mathcal{T}_{h}^{\Lambda}$ on the interface $\left.\Gamma_{1}\right)$.

At the spatial level, we introduce nested finite element approximation spaces $\widetilde{\mathcal{U}}_{H}$ and $\mathcal{W}_{h}^{s}$ for $s=1, \ldots, 4$. As illustrated in Figure 3, we use regular meshes $\mathcal{T}_{H}^{\widetilde{\Omega}}$ and $\mathcal{T}_{h}^{\Lambda_{s}}$ composed of triangular elements of respective sizes $H=0.25$ and $h=0.025$ in $\widetilde{\Omega}$ and $\Lambda_{s}$, leading to approximation spaces $\widetilde{\mathcal{U}}_{H}$ and $\mathcal{W}_{h}^{s}(s=1, \ldots, 4)$ with dimensions 441 and 1681 respectively.

At the stochastic level, we introduce for the approximation space $\mathcal{S}_{P}$ a polynomial space with total degree $p=3$ in 4 dimensions (using Legendre polynomial chaos basis). It yields a dimension $\operatorname{dim}\left(\mathcal{S}_{P}\right)=35$. 


\subsection{Convergence analysis}

We denote by $U \in \widetilde{\mathcal{U}}_{H} \otimes \mathcal{S}_{P}$ the reference global solution obtained by a direct resolution of the problem, and by $U_{n} \in \widetilde{\mathcal{U}}_{H} \otimes \mathcal{S}_{P}$ the global iterates obtained with the global-local iterative algorithm. We introduce the error indicator

$$
\varepsilon_{\Omega}\left(U_{n} ; U\right)=\frac{\left\|U-U_{n}\right\|_{0, \Omega}}{\|U\|_{0, \Omega}}
$$

with $\|\cdot\|_{0, \Omega}$ the natural norm on $L_{P_{\xi}}^{2}\left(\Xi ; L^{2}(\Omega)\right)$ defined by

$$
\|u\|_{0, \Omega}^{2}=\int_{\Xi} \int_{\Omega(y)} u(x, y)^{2} d x d P_{\xi}(y) .
$$

We first analyze the influence of relaxation parameter $\rho$ on the convergence of the iterative algorithm. The optimal relaxation parameter of the global-local iterative algorithm can be shown to be (see [34])

$$
\rho^{o p t}=\frac{2}{\lambda_{\min }(A)+\lambda_{\max }(A)}
$$

where $\lambda_{\min }(A)$ and $\lambda_{\max }(A)$ are the lowest and largest eigenvalues of operator $A(V)=V-\Phi(\Psi(V), V)$, defined in (18). In practice, $\lambda_{\max }(A)$ is estimated using some power iterations (typically 2 or 3 ), each power iteration requiring the solution of one local problem (for computing $\Psi(V)$ ) and one global problem (for computing $\Phi(\Psi(V), V)$ ). Since it is hard task to determine $\lambda_{\min }(A)$, in the following the optimal relaxation parameter is approximated as follows:

$$
\rho^{o p t} \approx \rho^{*}=\frac{1}{\lambda_{\max }(A)}
$$

This approximation is rather pertinent if the operator $A$ is well conditioned, that means $\lambda_{\min }(A)$ and $\lambda_{\max }(A)$ are of the same order.

The iterative global-local algorithm is carried out using fictitious coefficient $\widetilde{K}=1$ on $\widetilde{\Lambda}$ which corresponds to a global problem that does not see any heterogeneity. Figure 4 shows the error indicator $\varepsilon_{\Omega}\left(U_{n} ; U\right)$ with respect to the number of iterations for $\alpha \in\{-0.9,10,30,100\}$ and for different values of relaxation parameter $\rho$. We observe in all cases that the approximate optimal relaxation parameter $\rho^{*}$ gives almost optimal convergence, the optimal convergence being actually obtained for the true optimal relaxation parameter $\rho^{o p t}$. We observe that the algorithm converges quite fast (relative error less than $10^{-5}$ for $n \leq 10$ ) for the different levels of heterogeneities.

Figure 5 gathers the previous convergence curves obtained with the respective approximate optimal relaxation parameters $\rho^{*}$ for $\alpha \in\{-0.9,10,30,100\}$ in order to show the effect of the amplitude of the diffusion coefficients in the patches on the convergence of the algorithm. We see that convergence deteriorates as $\alpha$ increases, that is to say as the ratio between the maximum value of the diffusion field $K_{s}$ and the fictitious field $\widetilde{K}$ increases. The question thus raises of the need to tune the fictitious field $\widetilde{K}$ to improve convergence. 


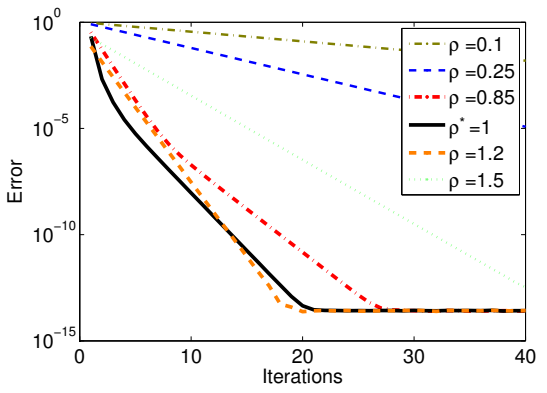

(a) $\alpha=-0.9$

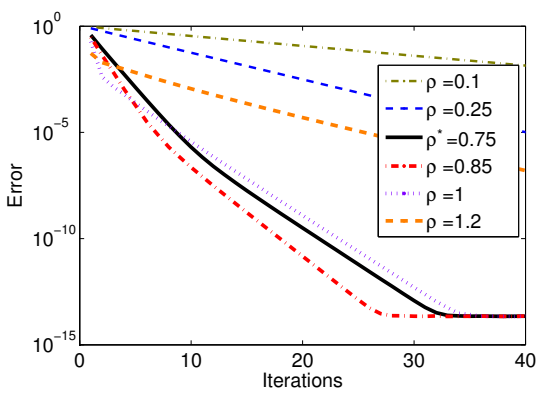

(c) $\alpha=30$

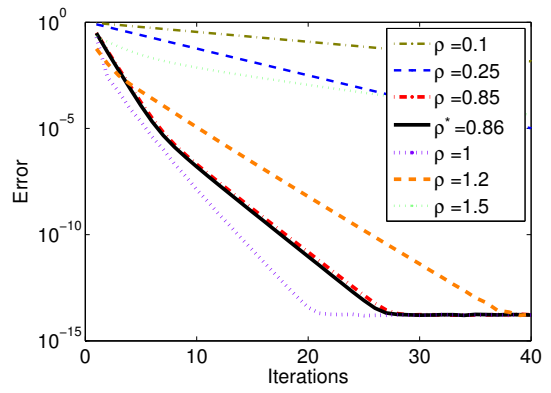

(b) $\alpha=10$

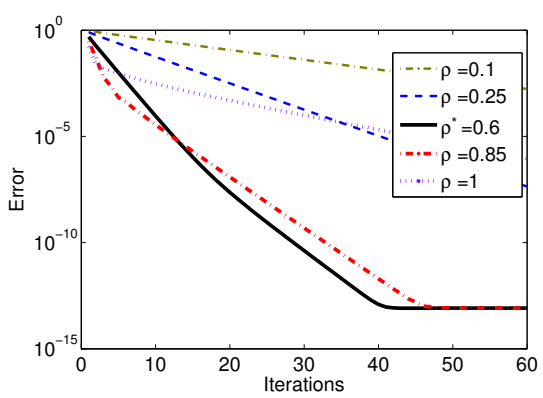

(d) $\alpha=100$

Figure 4: Evolution of the error indicator $\varepsilon_{\Omega}\left(U_{n} ; U\right)$ with the number of iterations $n$ for $\alpha \in\{-0.9,10,30,100\}$ and for different values of relaxation parameter $\rho$.

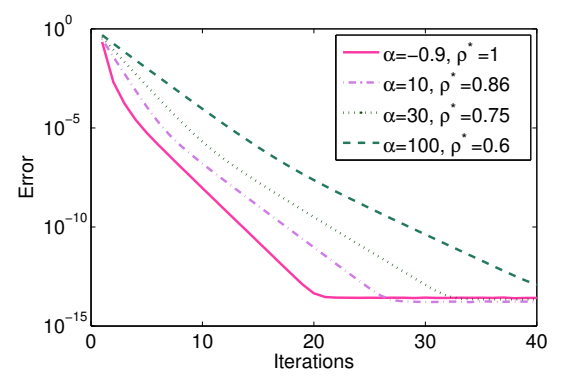

Figure 5: Evolution of the error indicator $\varepsilon_{\Omega}\left(U_{n} ; U\right)$ with the number of iterations $n$ for several values of parameter $\alpha$.

Noting that $\widetilde{K}$ acts as preconditioner of the iterative algorithm, we can study its influence on the convergence of the algorithm. We thus introduce a fictitious diffusion field of the form

$$
\widetilde{K}_{s}\left(x, \xi_{s}\right)=1+\beta k_{s}(x) \text { for } x \in \widetilde{\Lambda}_{s}, s \in\{1, \ldots, 4\},
$$

with $\beta \in \mathbb{R} . \beta=0$ corresponds to the case $\widetilde{K}=1$ studied earlier where the global problem does not see any heterogeneity, and $\beta=0.5$ corresponds to 
choosing the expectation of the diffusion field as the fictitious field in the global problem.

Figure 6 illustrates the convergence of iterates $U_{n}$ towards the reference solution $U$, for $\alpha \in\{-0.9,10,30,100\}$ and for different values of $\beta$. For each of these values, $u$ is computed using either the corresponding approximate optimal relaxation parameters $\rho^{*}$ (solid lines) or $\rho=1$ (dashed lines). On the one hand, as can be observed for $\alpha=100$, the global-local iterative algorithm using $\rho=1$ suffers from high heterogeneities when $\beta$ is set to 0 . Good convergence can be recovered by simply increasing $\beta$ up to 0.3 , or at least no more than 0.5 . On the other hand, it comes out that using the approximate optimal relaxation parameter ensures a good convergence rate whatever the value of $\beta$ in $[0,0.5]$ and whatever the level of heterogeneity. Finally, performing the local-global iteration algorithm with $\beta=0$ and $\rho=\rho^{*}$ is retained as the convenient choice for it gives good convergence properties and leads to a simple global problem with no heterogeneities.

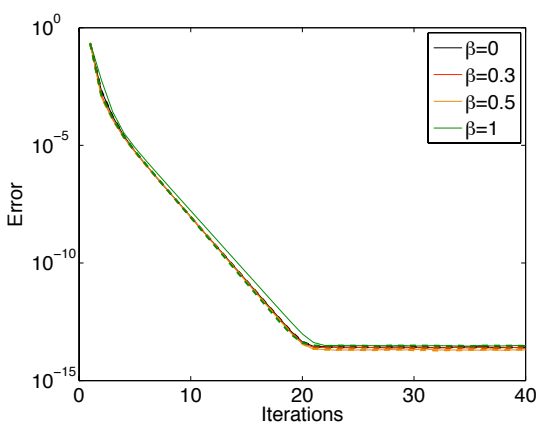

(a) $\alpha=-0.9$

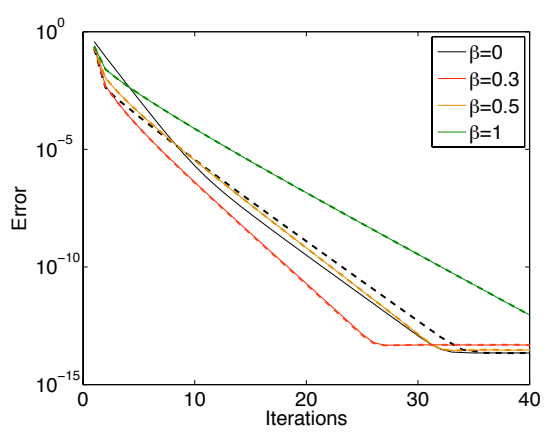

(c) $\alpha=30$

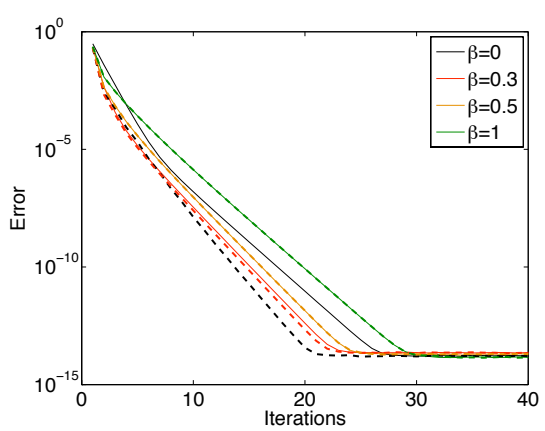

(b) $\alpha=10$

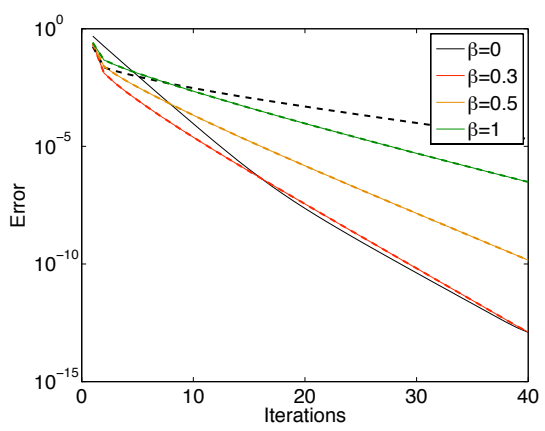

(d) $\alpha=100$

Figure 6: Evolution of the error indicator $\varepsilon_{\Omega}\left(U_{n} ; U\right)$ with the number of iterations $n$ of the local-global iterative algorithm for several values of $\beta$ and with $\rho=1$ (dashed lines) or $\rho^{*}=\frac{1}{\lambda_{\max }(A)}$ (solid lines). 


\subsection{Robustness with respect to approximations}

Global problems, involving a deterministic operator, are solved at the machine precision while local problems are solved using a Conjugate Gradient Squared (CGS) algorithm with a prescribed tolerance. Figure 7(a) shows the convergence of solution $U_{n}$, computed with the global-local iterative algorithm for different tolerances of CGS, towards the reference solution $U$ computed at machine precision. The iterative algorithm converges until the error indicator $\varepsilon_{\Omega}\left(U_{n} ; U\right)$ stabilizes around the tolerance imposed to the CGS solver, thus showing the robustness of the global-local iterative algorithm with respect to approximations.

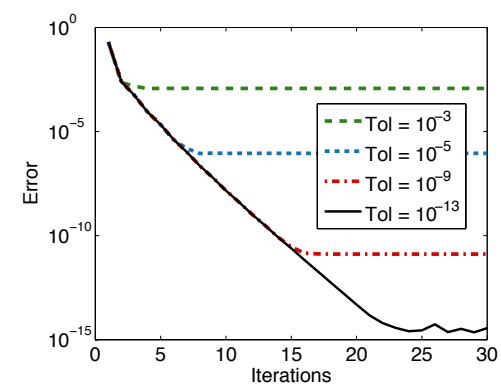

(a)

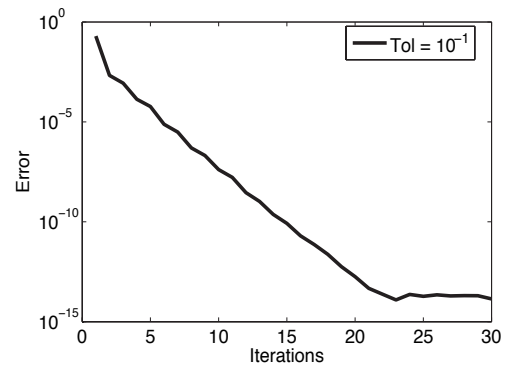

(b)

Figure 7: Evolution of error indicator $\varepsilon_{\Omega}\left(U_{n} ; U\right)$ with the number of iterations $n$ for $\alpha=$ $10, \tilde{K}=1, \rho=1$. (a) Local problems computed with a CGS algorithm with different tolerances and (b) local problems formulated on increments computed with a CGS algorithm with prescribed tolerance 0.1

The local problem is now formulated on the increment $\delta z_{n}=z_{n}-z_{n-1}$. A CGS algorithm with a coarse prescribed tolerance 0.1 is used to compute $\delta z_{n}$. In figure $7(\mathrm{~b})$, we see that $U_{n}$ converges to the reference solution down to the machine precision, as expected (see section 3.5).

\subsection{Illustration of probabilistic outputs}

This section aims at illustrating the statistics of the solution for further comprehension of the properties of the global-local iterative algorithm. Figure 8 shows the mean and the variance of the global solution $U$ and of the reconstructed solution $u . \mathbb{E}(Y)$ and $\mathbb{V}(Y)$ respectively denote the mean and variance of a random variable $Y$. The variance, which is here of rather small magnitude, is captured by the local solutions in the patches.

In order to quantify the impact of a random variable on the variance of the solution, we introduce the following sensitivity indices:

$$
S_{s}(u)(x)=\frac{\mathbb{V}\left(\mathbb{E}\left(u(x, \xi) \mid \xi_{s}\right)\right)}{\mathbb{V}(u(x, \xi))}, \quad \widetilde{S}_{s}(u)(x)=\frac{\mathbb{V}\left(\mathbb{E}\left(u(x, \xi) \mid \xi_{s}\right)\right)}{\sup _{y \in \Omega} \mathbb{V}(u(y, \xi))}
$$




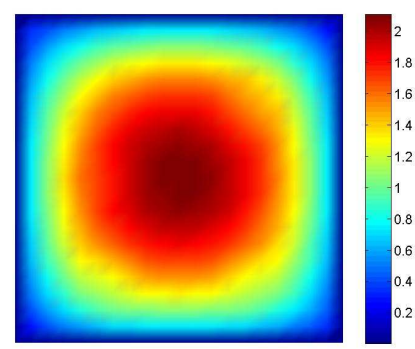

(a) $\mathbb{E}(U)$

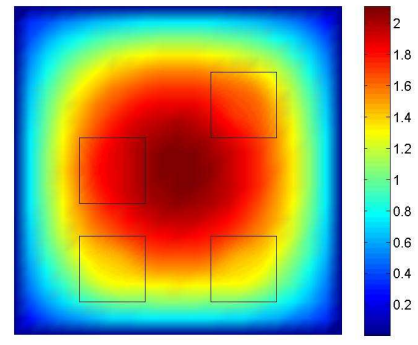

(c) $\mathbb{E}(u)$

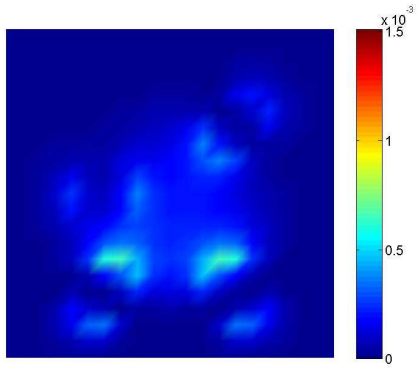

(b) $\mathbb{V}(U)$

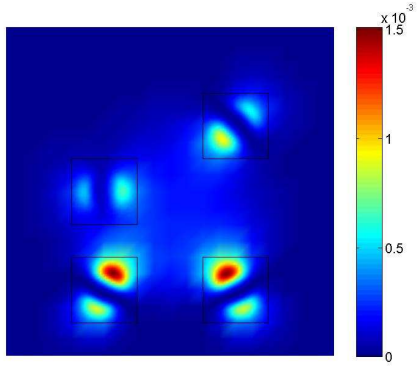

(d) $\mathbb{V}(u)$

Figure 8: Mean and variance of the global solution $U$ (a and b) and of the reconstructed solution $u$ (c and $\mathrm{d})$. Interface $\Gamma$ is displayed with black lines.

Note that $S_{s}(u)(x)$ are the classical first order Sobol indices such that $\sum_{s} S_{s}(u)(x)=$ 1 for all $x$, as it can be seen in figure 9 . The $\widetilde{S}_{s}(u)(x)$ are introduced to illustrate the zone of influence of a set of random variables $\xi_{s}$ (associated with a patch $\left.\Lambda_{s}\right)$ on the solution $u$. They are such that $\sum_{s} \widetilde{S}_{s}(u)(x)=\frac{\mathbb{V}(u(x, \xi))}{\sup _{y \in \Omega} \mathbb{V}(u(y, \xi))}$. The latter are plotted in figure 10 for the four patches. We can see that the solution is sensitive to a random variable $\xi_{s}$ essentially inside the respective patch $\Lambda_{s}$, for $s=1, \ldots, 4$. In this example, the impact of the input uncertainties on solution $u$ is localized and the sizes of the patches $\Lambda_{s}$ can be chosen to hold the main effects of the input random variables on the solution. 


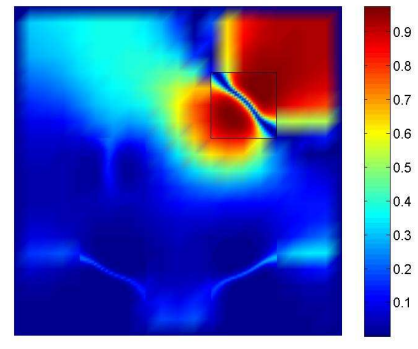

(a) $S_{1}(u)$

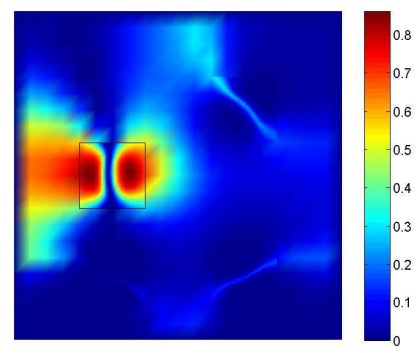

(c) $S_{3}(u)$

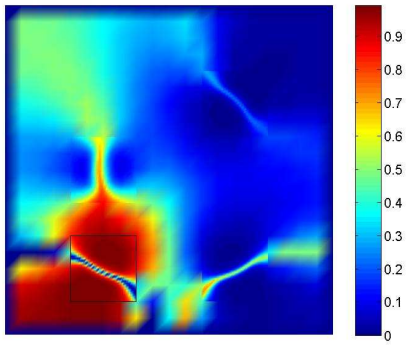

(b) $S_{2}(u)$

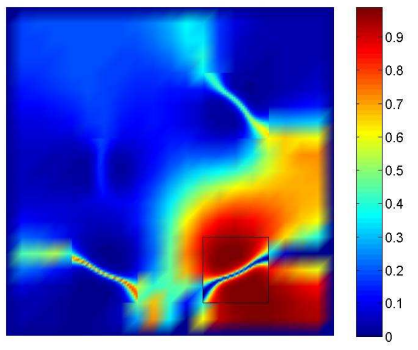

(d) $S_{4}(u)$

Figure 9: Sobol sensitivity indices $S_{s}(u)$ for $s=1,2,3,4$. 


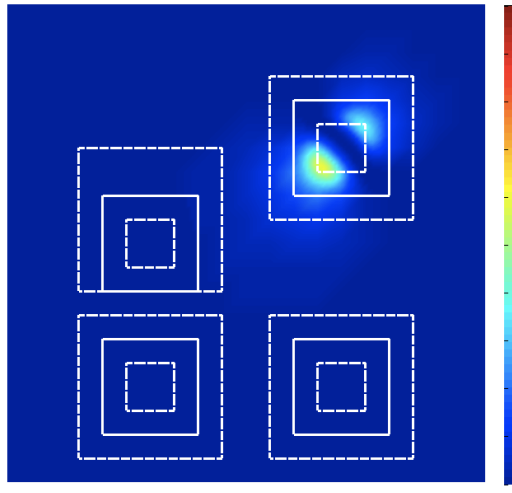

(a) $\widetilde{S}_{1}(u)$

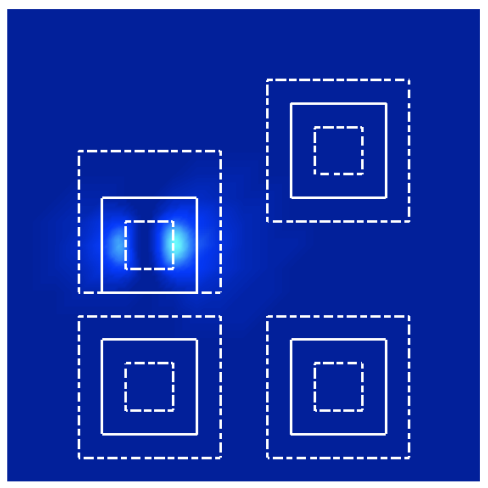

(c) $\widetilde{S}_{3}(u)$

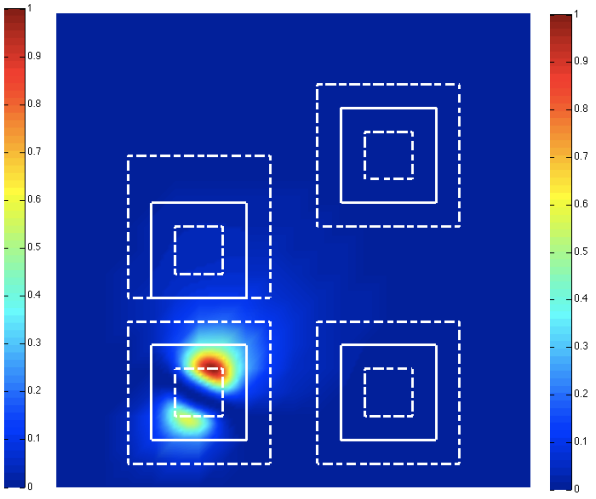

(b) $\widetilde{S}_{2}(u)$

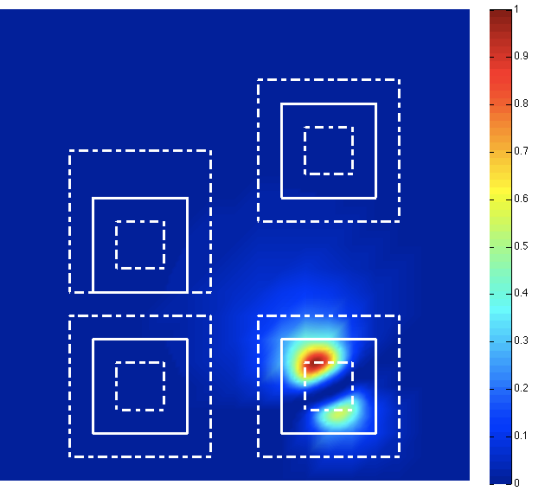

(d) $\widetilde{S}_{4}(u)$

Figure 10: Sensitivity indices $\widetilde{S}_{s}(u)$ for $s=1,2,3,4$. In white lines, interface $\Gamma$ for 3 different sizes of patches: $L=1$ in solid line and $L=0.5$ and $L=1.5$ in dashed line. 


\subsection{Motivations for the separation of scales}

Influence of the size of the patches on the convergence. In this section we study the influence of the size of the patches on the convergence of the global-local iterative algorithm. The algorithm is carried out for different side lengths $L \in$ $\{0.5,1,1.5\}$ of square patches $\Lambda_{s}$. For each size of the patch, the iterative algorithm is carried out using the approximate optimal relaxation parameter $\rho^{*}$. Figure 11 shows the convergence of $U_{n}$ towards $U$. The convergence rate appears to be independent of $L$ for $L \geq 1$, while it slightly deteriorates for $L=0.5$. Looking at figure 10 , we observe that for $L \geq 1$, the patches contain almost the entire zone of influence of random variables $\xi_{s}$, whereas for $L=0.5$, high values of the sensitivity index are observed outside the patches.

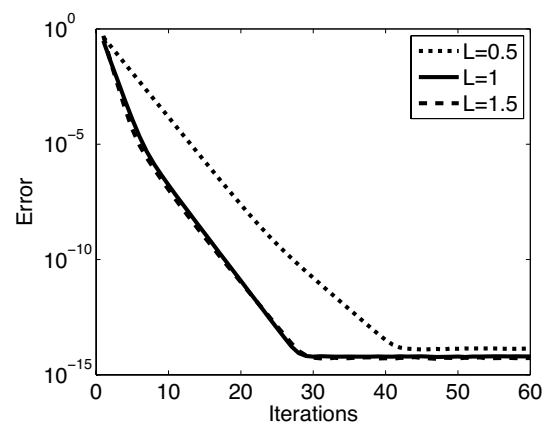

Figure 11: Convergence of the solution of the global-local iterative algorithm: evolution of the error indicator $\varepsilon_{\Omega}\left(U_{n} ; U\right)$ with the number of iterations $n$ for different side lengths $L$ of the patches.

Spectral content of solutions. In the following section, tensor approximation methods will be introduced in order to drastically reduce the costs for computing local and global problems. The performance of these tensor decomposition methods are strongly related to the spectral contents of the functions to be approximated. To that purpose, we now study the influence of the size of the patches on the rank of the Karhunen-Loève decompositions of the solutions. Let $u_{m}, U_{n, m}$ and $w_{n, m}^{s}$ be the rank- $m$ Karhunen-Loève decompositions of $u, U_{n}$ and $w_{n}^{s}$ respectively. Figure 12 shows the evolutions with respect to rank $m$ of $\varepsilon_{\Omega}\left(u_{m} ; u\right), \varepsilon_{\Omega}\left(U_{60, m} ; U_{60}\right)$ and $\varepsilon_{\Lambda_{s}}\left(w_{60, m}^{s} ; w_{60}^{s}\right)$ for $s=1, \ldots, 4$. For a given precision, the separation of scales operated by the global-local iterative algorithm allows to obtain iterates $U_{n}$ and $w_{n}^{s}$ that can be decomposed with a rank lower than the rank of the overall solution $u$. The convergence with $m$ of the error indicator $\varepsilon_{\Omega}\left(U_{60, m} ; U_{60}\right)$, which reflects the convergence of the Karhunen-Loève decomposition, is plotted in figure $12(\mathrm{~d})$ for different patch sizes $L=0.5,1,1.5$. We observe that increasing the size of the patches decreases the rank of the decompositions of the global solutions (for a fixed precision). This observation is in good agreement with that made earlier: choosing a sufficiently large patch that contains almost the entire zone of influence of the heterogeneities benefits 
both the convergence of the global-local iterative method and the spectral decomposition of the solutions of the iterative algorithm. By this splitting of the solution $u$ into a global solution $U$ and local solutions $w^{s}$, we obtain a fast decay in the spectrum of the functions to be approximated. That will be exploited by the following tensor approximation methods.

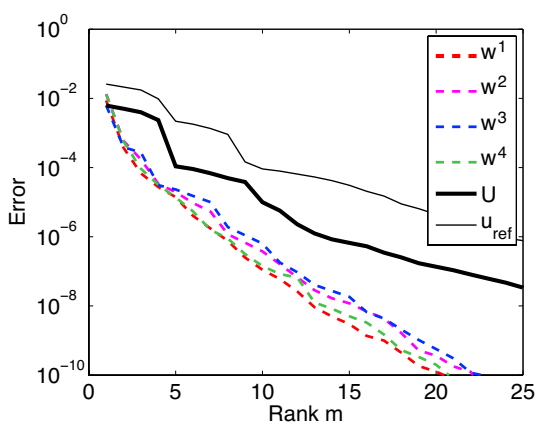

(a) $L=0.5$

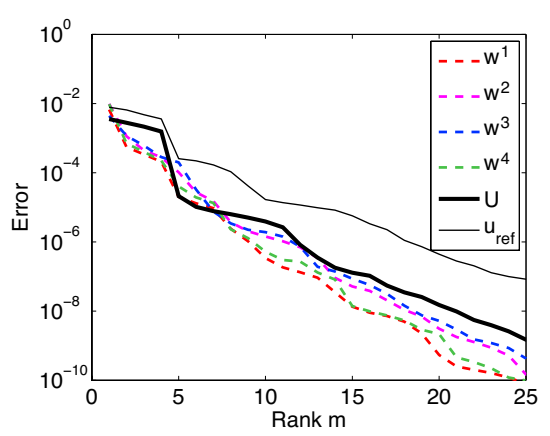

(c) $L=1.5$

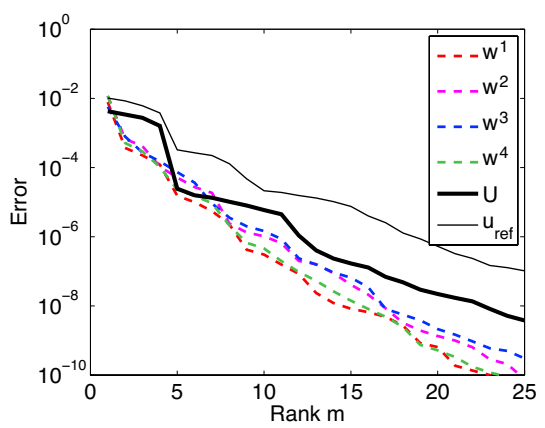

(b) $L=1$

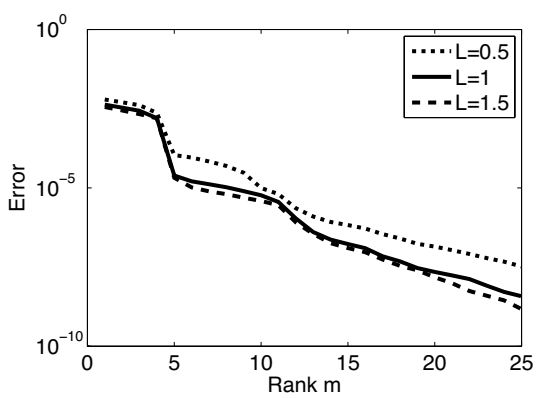

(d) $\varepsilon_{\Omega}\left(U_{m} ; U\right)$

Figure 12: Karhunen-Loève decompositions of $u$ and of the iterates $U_{n}$ and $w_{n}^{s}$ at iteration $n=60$. Evolutions with rank $m$ of $\varepsilon_{\Omega}\left(u_{m} ; u\right), \varepsilon_{\Omega}\left(U_{60, m} ; U_{60}\right)$ and $\varepsilon_{\Lambda_{s}}\left(w_{60, m}^{s} ; w_{60}^{s}\right)$ for $s=1, \ldots, 4$ and different dimensions of the patches: (a) $L=0.5$, (b) $L=1$ and (c) $L=1.5$.

(d) Evolution with rank $m$ of $\varepsilon_{\Omega}\left(U_{60, m} ; U_{60}\right)$ for the three dimensions of the patches.

\section{Solution of local and global problems using tensor approximation methods}

\subsection{Tensor product structure of stochastic function spaces}

If $\mathcal{H}$ is a deterministic function space, then

$$
\mathcal{H}^{\Xi}=\mathcal{H} \otimes \mathcal{S}
$$

with $\mathcal{S}=L_{P_{\xi}}^{2}(\Xi)$. An element in $\mathcal{H}^{\Xi}$ can then be interpreted as a two-order tensor. When the set of random variables $\xi$ is a collection of $N_{\Lambda}$ independent 
sets $\xi_{s}$ of random variables, then probability measure $P_{\xi}=P_{\xi_{1}} \otimes \ldots \otimes P_{\xi_{N_{\Lambda}}}$, and the space $\mathcal{S}$ has the following tensor product structure:

$$
\mathcal{S}=\mathcal{S}_{1} \otimes \ldots \otimes \mathcal{S}_{N_{\Lambda}}
$$

with $\mathcal{S}_{s}=L_{P_{\xi_{s}}}^{2}\left(\Xi_{s}\right)$. This tensor product structure of stochastic function spaces will be here exploited.

\subsection{Tensor spaces and tensor approximations}

For a tensor Hilbert space $V=V_{1} \otimes \ldots \otimes V_{d}$, we denote by $\mathcal{R}_{1}(V)$ the set of rank-one elements: $\mathcal{R}_{1}(V)=\left\{\otimes_{k=1}^{d} v^{k} \in V ; v^{k} \in V_{k}, 1 \leq k \leq d\right\}$, and by $\mathcal{R}_{m}(V)$ the set of rank- $m$ elements: $\mathcal{R}_{m}(V)=\left\{\sum_{i=1}^{m} v_{i} ; v_{i} \in \mathcal{R}_{1}(V), 1 \leq i \leq m\right\}$. For the case of function space $\mathcal{H}^{\Xi}$, with $\mathcal{H}$ a Hilbert space, different types of approximations of an element $u \in \mathcal{H}^{\Xi}$ can be obtained by using different structures of this tensor space. The reader can refer to [35, 36, 25] for an overview of tensor based numerical methods.

2-order canonical decomposition. Considering $\mathcal{H}^{\Xi}=\mathcal{H} \otimes \mathcal{S}$, we can introduce a rank- $m$ decomposition $u_{m} \in \mathcal{R}_{m}(\mathcal{H} \otimes \mathcal{S})$ which reads

$$
u_{m}=\sum_{i=1}^{m} Z_{i} \otimes \phi_{i} \quad \text { with } Z_{i} \in \mathcal{H} \text { and } \phi_{i} \in \mathcal{S} .
$$

This decomposition does not circumvent the curse of dimensionality associated with the approximation of functions in $\mathcal{S}$.

$\left(N_{\Lambda}+1\right)$-order canonical decomposition. Considering $\mathcal{H}^{\Xi}=\mathcal{H} \otimes \mathcal{S}_{1} \otimes \ldots \otimes \mathcal{S}_{N_{\Lambda}}$, we can introduce a rank- $m$ decomposition $u_{m} \in \mathcal{R}_{m}\left(\mathcal{H} \otimes \mathcal{S}_{1} \otimes \ldots \otimes \mathcal{S}_{N_{\Lambda}}\right)$ which reads

$$
u_{m}=\sum_{i=1}^{m} Z_{i} \otimes \phi_{i}^{1} \otimes \ldots \otimes \phi_{i}^{N_{\Lambda}} \quad \text { with } Z_{i} \in \mathcal{H} \text { and } \phi_{i}^{s} \in \mathcal{S}_{s}
$$

Remark 5. Note that a rank-m representation can be seen as an approximation of an element $u \in \mathcal{H}^{\Xi}$ on a m-dimensional basis composed of stochastic functions $\left\{\phi_{i}\right\}_{i=1}^{m}$ which are such that $\phi_{i}(\xi)=\phi_{i}^{1}\left(\xi_{1}\right) \ldots \phi_{i}^{N_{\Lambda}}\left(\xi_{N_{\Lambda}}\right)$.

Hierarchical canonical decomposition. A hierarchical canonical representation of $u \in \mathcal{H} \otimes \mathcal{S}$ can also be introduced as follows:

$$
u_{m}=\sum_{i=1}^{m} Z_{i} \otimes \phi_{i}, \quad \text { with } Z_{i} \in \mathcal{H} \text { and } \phi_{i} \in \mathcal{R}_{r_{i}}\left(\mathcal{S}_{1} \otimes \ldots \otimes \mathcal{S}_{N_{\Lambda}}\right) .
$$

This hierarchical canonical representation can be seen as an approximation of the two-order canonical decomposition (33). With sufficiently accurate rank$r_{i}$ approximations $\phi_{i}$, the best rank- $m$ approximation (35) is close to the best rank- $m$ approximation (33), which is far better than the decomposition (34) in 
most practical applications. Hereafter, we introduce the following notations for hierarchical representations:

$$
{ }_{H} \mathcal{R}_{1}^{r}\left(\mathcal{H}^{\Xi}\right)=\left\{Z \otimes \phi ; Z \in \mathcal{H}, \phi \in \mathcal{R}_{r}\left(\mathcal{S}_{1} \otimes \ldots \otimes \mathcal{S}_{N_{\Lambda}}\right)\right\} \subset \mathcal{R}_{1}(\mathcal{H} \otimes \mathcal{S}),
$$

and

$$
{ }_{H} \mathcal{R}_{m}^{r}\left(\mathcal{H}^{\Xi}\right)={ }_{H} \mathcal{R}_{1}^{r_{1}}\left(\mathcal{H}^{\Xi}\right)+\ldots+{ }_{H} \mathcal{R}_{1}^{r_{m}}\left(\mathcal{H}^{\Xi}\right) \subset \mathcal{R}_{m}(\mathcal{H} \otimes \mathcal{S})
$$

\subsection{A greedy algorithm for tensor decomposition (Proper Generalized Decom-} position)

We now present a methodology for the progressive construction of a tensor decomposition of the solution of a variational problem (global problem or local problems), under the form (33), (34) or (35). Let us consider a problem which admits the following generic variational form

$$
u \in \mathcal{H}^{\Xi}, \quad A(u, \delta u)=L(\delta u) \quad \forall \delta u \in \mathcal{H}^{\Xi},
$$

with $A$ symmetric continuous and coercive. The problem is equivalent to

$$
u=\arg \min _{v \in \mathcal{H}^{\Xi}} \mathcal{J}(v), \quad \mathcal{J}(v)=\frac{1}{2} A(v, v)-L(v) .
$$

We now present progressive constructions of decompositions of type (33), (34) or (35). Suppose that $u_{m-1}$ is known and denote by $\mathcal{X}$ the tensor approximation subset $\mathcal{R}_{1}(\mathcal{H} \otimes \mathcal{S}), \mathcal{R}_{1}\left(\mathcal{H} \otimes \mathcal{S}_{1} \otimes \ldots \otimes \mathcal{S}_{N_{\Lambda}}\right)$ or ${ }_{H} \mathcal{R}_{1}^{r_{m}}\left(\mathcal{H} \otimes \mathcal{S}_{1} \otimes \ldots \otimes \mathcal{S}_{N_{\Lambda}}\right)$ (respectively for decompositions of type (33), (34) or (35)). Then, we define a new element $v_{m} \in \mathcal{X}$ such that

$$
\mathcal{J}\left(u_{m-1}+v_{m}\right) \approx \min _{v \in \mathcal{X}} \mathcal{J}\left(u_{m-1}+v\right)
$$

where the symbol $\approx$ means that we compute an approximation of an optimal element in $\mathcal{X}$, and we define $u_{m}=u_{m-1}+v_{m}$.

Alternating minimization algorithms. In practice, we compute an approximation of $v_{m}$ using alternating minimization algorithms. For $\mathcal{X}=\mathcal{R}_{1}(\mathcal{H} \otimes \mathcal{S})$ (resp. $\left.\mathcal{X}=\mathcal{R}_{1}\left(\mathcal{H} \otimes \mathcal{S}_{1} \otimes \ldots \otimes \mathcal{S}_{N_{\Lambda}}\right)\right)$, we obtain an element $v_{m}=Z_{m} \otimes \phi_{m}$ (resp. $\left.v_{m}=Z_{m} \otimes \phi_{m}^{1} \otimes \ldots \otimes \phi_{m}^{N_{\Lambda}}\right)$ by alternatively minimizing on $Z_{m} \in \mathcal{H}$ and $\phi_{m} \in \mathcal{S}$ (resp. $\left.Z_{m} \in \mathcal{H}, \phi_{m}^{1} \in \mathcal{S}_{1}, \ldots, \phi_{m}^{N_{\Lambda}} \in \mathcal{S}_{N_{\Lambda}}\right)$. For $\mathcal{X}={ }_{H} \mathcal{R}_{1}^{r_{m}}\left(\mathcal{H} \otimes \mathcal{S}_{1} \otimes \ldots \otimes \mathcal{S}_{N_{\Lambda}}\right)$, we obtain an approximation $v_{m}=Z_{m} \otimes \phi_{m}$ by an alternating minimization algorithm which consists in solving alternatively:

- a minimization problem on $\mathcal{H}$ :

$$
Z_{m}=\arg \min _{Z \in \mathcal{H}} \mathcal{J}\left(u_{m-1}+Z \otimes \phi_{m}\right)
$$


- a minimization problem on $\mathcal{S}$ :

$$
\phi_{m} \approx \arg \min _{\phi \in \mathcal{S}} \mathcal{J}\left(u_{m-1}+Z_{m} \otimes \phi\right)
$$

where the symbol $\approx$ means that this problem is approximated using a second tensor approximation algorithm, leading to a rank- $r_{m}$ approximation $\phi_{m, r_{m}} \in \mathcal{R}_{1}^{r_{m}}\left(\mathcal{S}_{1} \otimes \ldots \otimes \mathcal{S}_{N_{\Lambda}}\right)$ of $\phi_{m}$, with a rank $r_{m}$ depending on the required precision. This approximation $\phi_{m, r_{m}}$ is constructed progressively by letting $\phi_{m, 0}=0$ and by defining $\phi_{m, r}=\phi_{m, r-1}+\varphi_{m, r}$, with $\varphi_{m, r} \in \mathcal{R}_{1}\left(\mathcal{S}_{1} \otimes \ldots \otimes \mathcal{S}_{N_{\Lambda}}\right)$ such that

$$
\varphi_{m, r} \in \arg \min _{\varphi \in \mathcal{R}_{1}\left(\mathcal{S}_{1} \otimes \ldots \otimes \mathcal{S}_{N_{\Lambda}}\right)} \mathcal{J}\left(u_{m-1}+Z_{m} \otimes\left(\phi_{m, r-1}+\varphi\right)\right) .
$$

These minimization problems on $\mathcal{R}_{1}\left(\mathcal{S}_{1} \otimes \ldots \otimes \mathcal{S}_{N_{\Lambda}}\right)$ are again solved with an alternating minimization algorithm consisting in minimizing successively on spaces $\mathcal{S}_{s}, 1 \leq s \leq N_{\Lambda}$. The reader can refer to [26] for practical implementation of these algorithms.

\subsection{Strategies for the resolution of the local and global problems}

The global problems (10) and local problems (11) within the global-local iterative algorithm can be formulated either on the iterates or on the increments between two iterates, as mentioned in section 3.5. It yields to different strategies.

First strategy: formulation on the iterates. The local and global problems are formulated on the iterates. Local problems are solved using tensor approximation methods, with a given tolerance $\epsilon$. Global problems are solved exactly or using the greedy algorithm for tensor decomposition, depending on wether the operator is deterministic or not. This corresponds to $\epsilon^{*}=0$ and $\epsilon>0$ in theorem 5. The error on the solution then tends to the given finite precision of the tensor decompositions.

Second strategy: formulation on the increments between iterates. Another possibility is to solve the local problems reformulated on the increments using tensor approximation methods. The greedy algorithm is used for the construction of a tensor decomposition of the increment, either with a fixed coarse tolerance $\epsilon^{*}$ or with a given low rank. Note that in the latter case, the effective precision $\epsilon^{*}$ of the resulting tensor decomposition depends on the iterate $n$ and can deteriorate with $n$. At each iteration, the solutions are truncated with a tolerance $\epsilon$ in order to maintain low rank decompositions (using classical tensor approximation algorithms $[35,25])$.

\section{Numerical example using tensor approximation methods}

\subsection{Description of the problem}

We consider diffusion problem (1) with $f=1, \Gamma_{D}=\partial \Omega(\xi)$ and $\Gamma_{N}=\emptyset$. We define seven patches $\Lambda_{s}\left(\xi_{s}\right), s=1, \ldots, 7$, represented in figure 13 with 
side length $L=2$ and respective centers $c_{s}: c_{1}=(2,2), c_{2}=(5,2), c_{3}(8,2)$, $c_{4}=(2,6), c_{5}=(5,8), c_{6}=(8,6)$ and $c_{7}=(1,9)$.

The diffusion coefficient is uncertain on patches $\Lambda_{1}, \Lambda_{3}$ and $\Lambda_{5}$. We consider

$$
K= \begin{cases}1 & \text { for } x \in \Omega \backslash\left(\Lambda_{1} \cup \Lambda_{3} \cup \Lambda_{5}\right) \\ 1+\sum_{k=1}^{5} \xi_{s}^{k} k_{s}^{k}(x) & \text { for } x \in \Lambda_{s}, \text { for } s \in\{1,3\} \\ 1+\xi_{5} I(x) & \text { for } x \in \Lambda_{5}\end{cases}
$$

with

$$
k_{s}(x)=10 \exp \left(-2 \frac{\left|x-a_{s}^{k}\right|^{2}}{0.25^{2}}\right), s \in\{1,3\}
$$

and

$$
I(x)= \begin{cases}3 & \text { if } x \in D_{5}=(4.5,5.5) \times(7.5,8.5) \\ 0 & \text { if } x \in \Lambda_{5} \backslash D_{5}\end{cases}
$$

where $a_{s}^{k}$, for $s \in\{1,3\}$ and for $k \in\{1, \ldots, 5\}$, is a point in patch $\Lambda_{s}$ and where the $\xi_{1}^{k}, \xi_{3}^{k}$ and $\xi_{5}$ are independent uniform random variables on $\Xi_{1}^{k}=(0,1)$, $\Xi_{3}^{k}=(0,1)$ and $\Xi_{5}=(0,1)$ respectively. We denote $\Xi_{1}=\times_{k=1}^{5} \Xi_{1}^{k} \subset \mathbb{R}^{5}$, $\Xi_{3}=\times_{k=1}^{5} \Xi_{2}^{k} \subset \mathbb{R}^{5}$ and $\Xi_{5} \subset \mathbb{R}$ the sets of elementary events associated with patches $\Lambda_{1}=\widetilde{\Lambda}_{1}, \Lambda_{3}=\widetilde{\Lambda}_{3}$ and $\Lambda_{5}=\widetilde{\Lambda}_{5}$ respectively.

Domain $\Omega(\xi)$ also presents geometrical details with homogeneous Dirichlet conditions on circular internal boundaries. Let us denote $s_{D}=\{2,4,6,7\}$ the set of indices of the patches with random internal Dirichlet boundaries. Patches $\Lambda_{2}(\xi)$ and $\Lambda_{4}(\xi)$ present circular boundaries of random radii $r_{s}=0.3+0.1 \xi_{s}$ and centered on $c_{s}$ for $s=2,4$. In patch $\Lambda_{6}(\xi)$ we define a circular boundary with radius $r_{6}=0.3$ and with random position of the center $c\left(\xi_{6}\right)=\left(8+0.1 \xi_{6}, 6\right)$. Finally, in patch $\Lambda_{7}(\xi)$ we consider a corner in a circular arc with center $(1+$ $\left.0.5 \xi_{7}, 9-0.5 \xi_{7}\right)$ and with radius $r_{7}=1+0.5 \xi_{7}$. $\xi_{s}$ for $s \in s_{D}$ are uniform identically distributed random variables on $\Xi_{s}=(0,1)$ respectively.

We introduce fictitious patches $\widetilde{\Lambda}_{2}, \widetilde{\Lambda}_{4}, \widetilde{\Lambda}_{6}$ and $\widetilde{\Lambda}_{7}$ and fictitious domain $\widetilde{\Omega}$ such that $\widetilde{\Omega}=(0,10) \times(0,10)$ and $\Omega(\xi)=\left(\widetilde{\Omega} \backslash \cup_{s \in s_{D}} \widetilde{\Lambda}_{s}\right) \cup_{s \in s_{D}} \Lambda_{s}\left(\xi_{s}\right)$.

The global problem is defined on domain $\widetilde{\Omega}$ which does not contain any geometrical detail and it is associated with deterministic diffusion coefficient $\widetilde{K}=1$ on $\widetilde{\Omega}$. The global problem has thus a deterministic operator. The local problems on $\Lambda_{s}(\xi)$ with $s \in s_{D}$ are solved using the characteristic function method. The characteristic functions $\psi^{s}\left(x, \xi_{s}\right)$ are chosen as the opposite of the random level-set functions the iso-zero of which in patch $\widetilde{\Lambda}_{s}$ (for $s \in s_{D}$ ) define the random boundaries inside $\widetilde{\Lambda}_{s}$. Non nested finite element approximation spaces $\widetilde{\mathcal{U}}_{H}$ and $\widetilde{\mathcal{W}}_{h}$ are introduced using regular meshes with element sizes $H=$ 0.5 and $h \approx 0.07$ on $\widetilde{\Omega}$ and $\widetilde{\Lambda}$ respectively. The dimensions of approximation spaces $\widetilde{\mathcal{U}}_{H}$ and $\widetilde{\mathcal{W}}_{h}^{s}$ (for $s=1, \ldots, 7$ ) are 441 and 900 respectively. We introduce a stochastic approximation space $\mathcal{S}_{P}=\mathcal{S}_{1, P_{1}} \otimes \ldots \otimes \mathcal{S}_{7, P_{7}}$, where for $s \in s_{D}$, the $\mathcal{S}_{s, P_{s}}$ are piecewise polynomial spaces with degree 3 on a uniform partition of $\Xi_{s}$ (resulting in dimensions $P_{s}=24$ for $s \in s_{D}$ ), and where for $s \notin s_{D}$, the $\mathcal{S}_{s, P_{s}}$ are multidimensional polynomial spaces of degree 3 (resulting in dimensions $P_{1}=P_{3}=56$ and $\left.P_{5}=4\right)$. It results in a dimension $\operatorname{dim}\left(\mathcal{S}_{P}\right) \approx 4.210^{9}$. 


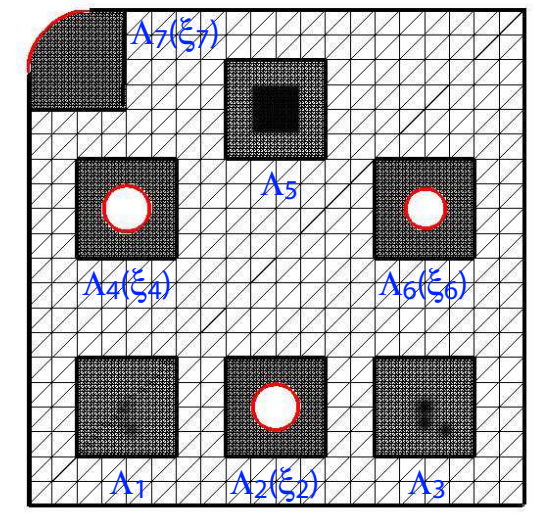

Figure 13: Diffusion problem with 7 patches

Global and local problems are approximated in canonical or hierarchical canonical tensor formats. Global problems are solved exactly since they only involve a deterministic operator, while the local problems are solved using the greedy constructions presented in section 7.3.

\subsection{Tensor approximations of the local solutions using Proper Generalized De- compositions}

\subsubsection{Canonical tensor approximations}

Here, canonical tensor approximations of the local solutions (in $\mathcal{R}_{m}\left(\widetilde{\mathcal{W}}_{h}^{s} \otimes\right.$ $\left.\mathcal{S}_{1, P_{1}} \otimes \ldots \otimes \mathcal{S}_{7, P_{7}}\right)$ ) are constructed using the greedy algorithm presented in section 7.3. When the local problem is formulated on the iterate $z_{n}$, we solve the problem with a prescribed tolerance $\epsilon=5 \cdot 10^{-3}$ on the residual. When the local problem is formulated on the increment $\delta z_{n}$, we use a coarse tolerance $\epsilon^{*}=0.1$ on the residual. At each iteration the global iterates $U_{n}$ and the Lagrange multipliers $\lambda_{n}^{s}$ are compressed using classical canonical tensor decomposition algorithms in order to reduce their representation rank. For this compression step, we use a tolerance $\epsilon=5 \cdot 10^{-3}$.

In order to illustrate the robustness of the method with respect to approximations, the error of stagnation $\varepsilon_{\widetilde{\Omega}}\left(U_{n-1} ; U_{n}\right)$ is plotted as a function of the iteration $n$ in figure 14. The relaxation parameter of the global-local iterative algorithm is chosen as $\rho=0.2 \approx \rho^{*}=\frac{1}{\lambda_{\max }(A)}$. We see that for both formulations, on the solution or on the increment, the error decreases down to the finite precision $5.10^{-3}$ introduced in the tensor decompositions. Note that when formulating local problems on increments, we still observe a stagnation of the global-local algorithm due to additional errors introduced in the compression of the global iterates and the Lagrange multipliers (corresponding to an error of type $\epsilon$ in theorem 5). 


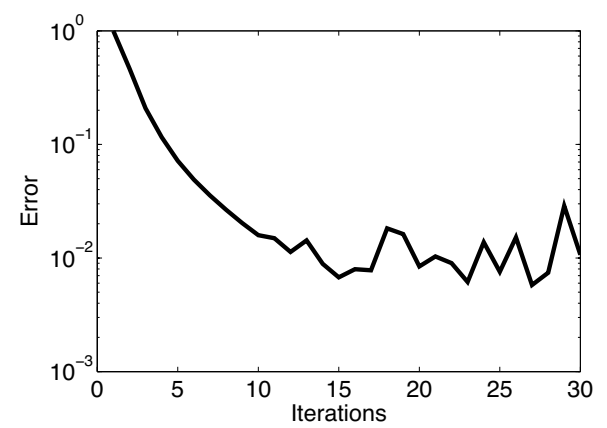

(a) Formulation on the solution $z_{n}$.

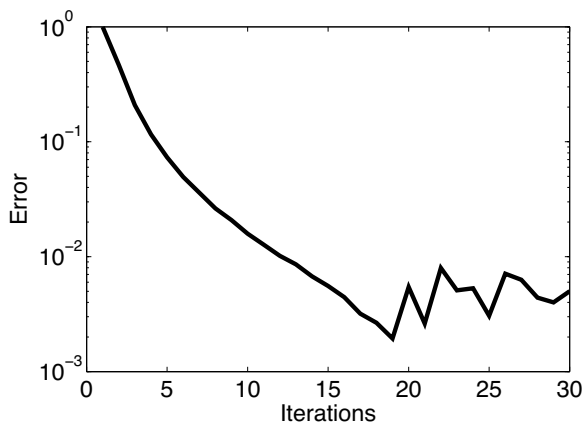

(b) Formulation on the increment $\delta z_{n}$.

Figure 14: Evolution of error indicator $\varepsilon_{\widetilde{\Omega}}\left(U_{n-1}, U_{n}\right)$ with the number of iterations $n$ with a prescribed tolerance $\epsilon=5.10^{-3}$ on the canonical representation of global solutions. (a) Formulation on the solution $z_{n}$, canonical decomposition with prescribed tolerance $\epsilon=5.10^{-3}$, (b) formulation on the increment $\delta z_{n}$, canonical decomposition with prescribed tolerance $\epsilon^{*}=0.1$.

Figure 15 shows a random sample of the solutions obtained at iteration $n=14$. For a sake of clarity, the subscript $n$ referring to the iteration has been omitted in the legend of the figure and $v_{m}$ denotes here a rank- $m$ decomposition of a quantity $v$. This light notation is adopted in the following of this section only. We see that the local solutions in patches with random Dirichlet boundaries have higher ranks than those in patches with random diffusion coefficients.

We now analyze the convergence of rank- $m$ canonical tensor decompositions $U_{m}$ and $w_{m}^{s}$ of global iterate $U$ and local iterates $w^{s}$ at iteration $n=14$. Figure 16 shows the evolution of $\varepsilon_{\widetilde{\Omega}}\left(U_{m} ; U\right)$ and $\varepsilon_{\widetilde{\Lambda}_{s}}\left(w_{m}^{s} ; w^{s}\right)$ with respect to rank $m$. The local solutions in patches with random geometry still have higher ranks than those of $U$ and of the other local solutions in patches with random diffusion fields. However, by operating a separation of scales, the multiscale method with patches has participated in reducing the ranks of the tensor decompositions of the local and global solutions and therefore contributes to the good performance of the overall method.

\subsubsection{Hierarchical canonical tensor approximations}

Now, hierarchical canonical tensor approximations are introduced for solving local problems, which are here formulated on the iterate (and not on the increment). Approximations in ${ }_{H} \mathcal{R}_{m}^{r}\left(\widetilde{\mathcal{W}}_{h}^{s} \otimes \mathcal{S}^{P}\right)$ are constructed using the greedy algorithm presented in section 7.3. The tolerance is set to $5 \cdot 10^{-3}$ in terms of the residual error at the two levels of approximations in the hierarchical canonical decomposition. At each iteration the global iterates $U_{n}$ are also compressed in ${ }_{H} \mathcal{R}_{m}^{r}\left(\widetilde{\mathcal{U}}_{H} \otimes \mathcal{S}^{P}\right)$ with a tolerance $\epsilon=5.10^{-3}$ at each level of approximation in the hierarchical canonical decomposition. Figure 17 illustrates the convergence of the global-local iterative algorithm and again we observe a very good con- 


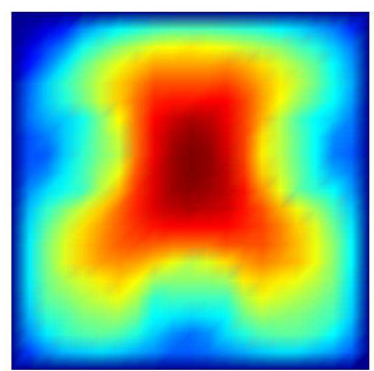

(a) $U_{5}$

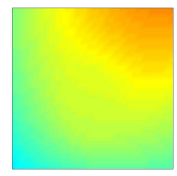

(c) $w_{8}^{1}=U^{\diamond}+z_{3}^{1}$

(d) $w_{27}^{2}=U^{\diamond}+$
$\psi^{2} z_{11}^{2}$
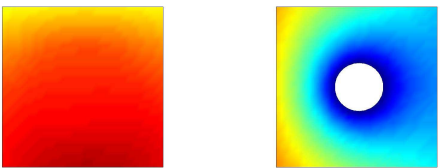

(e) $w_{8}^{3}=U^{\diamond}+z_{3}^{3}$
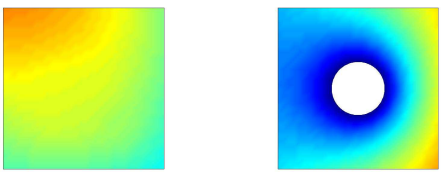

(f) $w_{25}^{4}=U^{\diamond}+$ $\psi^{4} z_{10}^{4}$
(g) $w_{9}^{5}=U^{\diamond}+z_{4}^{5}$
(h) $w_{23}^{6}=U^{\diamond}+\psi^{6} z_{9}^{6}$
(i) $w_{17}^{7}=U^{\diamond}+\psi^{7} z_{6}^{7}$

Figure 15: Random samples of global solution $U$, local solutions $w^{s}$ for $s=1, \ldots, 7$ and reconstructed solution $u$ at iteration $n=14$.

vergence down to an error lower than the prescribed tolerance $5 \cdot 10^{-3}$. This is due to the fact that hierarchical canonical decompositions converge very fast (as illustrated below) and that the effective precision of the resulting low rank decompositions is less than the prescribed tolerance.

Figure 18 shows the ranks $m$ of local solutions $z^{s}$ with respect to the iterations $n$ using either the canonical or the hierarchical canonical tensor approximations. The hierarchical decomposition shows a significant improvement in the rank of local solutions $z^{s}$. Indeed, while the canonical decompositions reach ranks up to $m=4$ in patches with random diffusion field or $m=12$ for patches with random Dirichlet boundaries, the hierarchical decompositions have very low ranks 1 or 2 . Having these low rank representations, the Lagrange multipliers $\lambda^{s}$ also have low ranks and do not need to be compressed for efficiency reasons. Therefore, no additional error is introduced. 


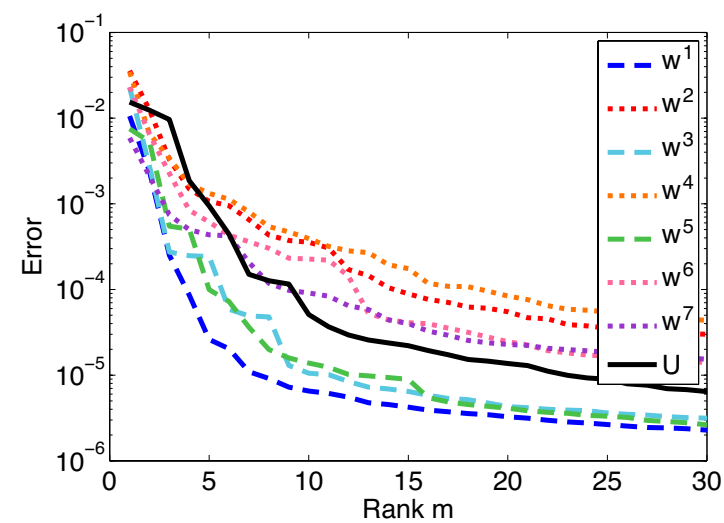

Figure 16: Evolutions of $\varepsilon_{\widetilde{\Omega}}\left(U_{m} ; U\right)$ and $\varepsilon_{\widetilde{\Lambda}_{s}}\left(w_{m}^{s} ; w^{s}\right)$ for $s=1, \cdots, 7$ with respect to rank $m$. $U_{m}$ and $w_{m}^{s}$ denote rank- $m$ canonical decompositions of solutions at iteration $n=14$. (Dashed lines) patches with random diffusion field and (Dotted lines) patches with random internal boundaries with Dirichlet conditions.

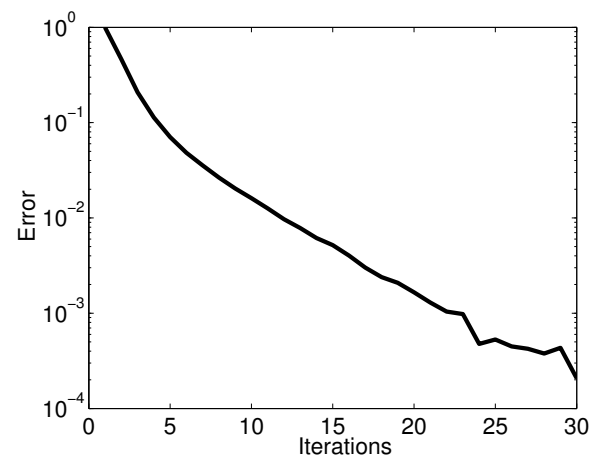

Figure 17: Evolution of error indicator $\varepsilon_{\widetilde{\Omega}}\left(U_{n-1}, U_{n}\right)$ with the number of iterations $n$ when using hierarchical canonical tensor approximations for local and global solutions (with a prescribed tolerance $\epsilon=5.10^{-3}$ ).

\section{Conclusion}

A dedicated method has been proposed for dealing with multiscale problems with localized sources of uncertainties. It is based on a domain decomposition method with patches associated with a global-local iterative algorithm that enables the introduction of refined local approximation to well describe local quantities. When dealing with geometrical variability, fictitious domain methods that enable the formulation of the problem on a tensor product space have been introduced. The global-local iterative algorithm has nice convergence properties. Indeed, results on the first numerical example have shown that the parameters of the algorithm can be chosen so to have simple deterministic global problems while conserving a good convergence rate. The separation of scales also benefits 


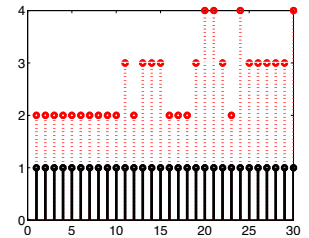

(a) $s=1$

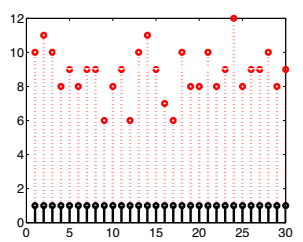

(b) $s=2$

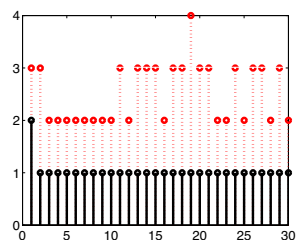

(c) $s=3$

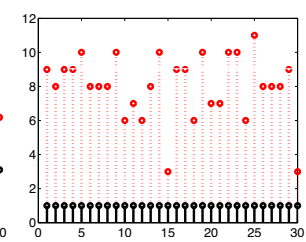

(d) $s=4$

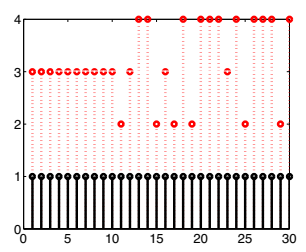

(e) $s=5$

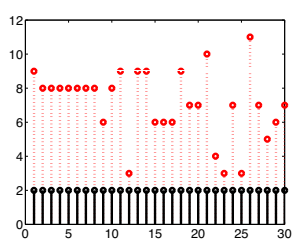

(f) $s=6$

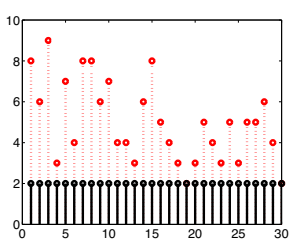

(g) $s=7$

Figure 18: Ranks of local solutions $z^{s}$ for patches $s=1, \ldots, 7$ : (red) canonical decomposition and (black) hierarchical canonical decomposition.

tensor based methods that are used to solve high dimensional global and local problems. The hierarchical canonical decomposition has revealed particularly efficient for obtaining very low ranks decompositions of the global and local solutions. The sizes of the patches should however be chosen with attention since they reveal to have a double impact: when chosen too small, that is to say when the patch does not hold the main effects of the localized uncertainties on the response, the convergence rate of the iterative algorithm deteriorates and the global solutions have higher rank decompositions.

The global and local problems can be simplified by exploiting the sensitivity indices introduced for assessing the zone of influence of the different sets of random variables on the solution. In fact, the dependancy of the global and local solutions to some sets of variables can be neglected for further computational savings. The proposed approach can be extended to a wider class of models including non-linear models.

\section{Acknowledgements}

This work is supported by the French National Research Agency (grant TYCHE ANR-2010-BLAN-0904).

\section{References}

[1] H. G. Matthies. Stochastic finite elements: Computational approaches to stochastic partial differential equations. ZAMM - Journal of Applied Mathematics and Mechanics, 88(11):849-873, 2008. 
[2] A. Nouy. Recent developments in spectral stochastic methods for the numerical solution of stochastic partial differential equations. Archives of Computational Methods in Engineering, 16(3):251-285, 2009.

[3] D. Xiu. Fast numerical methods for stochastic computations: a review. Communication in Computational Physics, 5(2-4):242-272, 2009.

[4] O. P. Le Maître and O. M. Knio. Spectral Methods for Uncertainty Quantification: With Applications to Computational Fluid Dynamics. Springer, 1st edition, 2010.

[5] A. Sarkar, N. Benabbou, and R. Ghanem. Domain decomposition of stochastic pdes: Theoretical formulations. International Journal for $\mathrm{Nu}$ merical Methods in Engineering, 77(5):689-701, 2009.

[6] X. F. Xu. A multiscale stochastic finite element method on elliptic problems involving uncertainties. Computer Methods in Applied Mechanics and Engineering, 196(2528):2723 - 2736, 2007.

[7] X. F. Xu, X. Chen, and L. Shen. A Green-function-based multiscale method for uncertainty quantification of finite body random heterogeneous materials. Computers and Structures, 87:1416-1426, 2009.

[8] B. Ganapathysubramanian and N. Zabaras. A stochastic multiscale framework for modeling flow through random heterogeneous porous media. Journal of Computational Physics, 228(2):591 - 618, 2009.

[9] X. Ma and N. Zabaras. A stochastic mixed finite element heterogeneous multiscale method for flow in porous media. Journal of Computational Physics, 230(12):4696 - 4722, 2011.

[10] E. Stein and S. Ohnimus. Coupled model- and solution-adaptivity in the finite-element method. Computer Methods in Applied Mechanics and Engineering, 150(14):327 - 350, 1997.

[11] A. Düster, A. Niggl, and E. Rank. Applying the hp-d version of the fem to locally enhance dimensionally reduced models. Computer Methods in Applied Mechanics and Engineering, 196(3740):3524 - 3533, 2007.

[12] J. M. Melenk and I. Babuška. The partition of unity method: basic theory and applications. Computer Methods in Applied Mechanics and Engineering, 39:289-314, 1996.

[13] N. Moës, J. Dolbow, and T. Belytschko. A finite element method for crack growth without remeshing. Int. J. for Numerical Methods in Engineering, 46:131-150, 1999.

[14] L. Gendre, O. Allix, P. Gosselet, and F. Comte. Non-intrusive and exact global/local techniques for structural problems with local plasticity. Computational Mechanics, 44(2):233-245, 2009. 
[15] L. Gendre, O. Allix, and P. Gosselet. A two-scale approximation of the schur complement and its use for non-intrusive coupling. International Journal for Numerical Methods in Engineering, 87:889-905, 2011.

[16] A. Lozinski. Méthodes numériques et modélisation pour certains problèmes multi-échelles. Université Paul Sabatier, Toulouse 3, 2010.

[17] A. Lozinski and O. Pironneau. Numerical zoom for localized multiscales. Numerical Methods for Partial Differential Equations, 27:197 - 207, 2011.

[18] C. Hager, P. Hauret, P. Le Tallec, and B. I. Wohlmuth. Solving dynamic contact problems with local refinement in space and time. Computer Methods in Applied Mechanics and Engineering, 201 - 204(0):25 - 41, 2012.

[19] F. Brezzi, J.-L. Lions, and O. Pironneau. Analysis of a chimera method. Comptes Rendus de l'Académie des Sciences - Series I - Mathematics, 332(7):655 - 660, 2001.

[20] G. J. Wagner and W. K. Liu. Coupling of atomistic and continuum simulations using a bridging scale decomposition. Journal of Computational Physics, 190(1):249 - 274, 2003.

[21] S.P. Xiao and T. Belytschko. A bridging domain method for coupling continua with molecular dynamics. Computer Methods in Applied Mechanics and Engineering, 193(17 - 20):1645 - 1669, 2004.

[22] H. Ben Dhia. Multiscale mechanical problems: the Arlequin method. Comptes Rendus de l'Académie des Sciences, Paris, Série IIb, 326:899904, 1998.

[23] L. Chamoin, J.T. Oden, and S. Prudhomme. A stochastic coupling method for atomic-to-continuum monte-carlo simulations. Computer Methods in Applied Mechanics and Engineering, 197(4344):3530 - 3546, 2008.

[24] R. Cottereau, D. Clouteau, H. Ben Dhia, and C. Zaccardi. A stochasticdeterministic coupling method for continuum mechanics. Computer Methods in Applied Mechanics and Engineering, 200(4748):3280 - 3288, 2011.

[25] W. Hackbusch. Tensor Spaces and Numerical Tensor Calculus, volume 42 of Series in Computational Mathematics. Springer, 2012.

[26] A. Nouy. Proper Generalized Decompositions and separated representations for the numerical solution of high dimensional stochastic problems. Archives of Computational Methods in Engineering, 17(4):403-434, 2010.

[27] M. Chevreuil and A. Nouy. Model order reduction based on proper generalized decomposition for the propagation of uncertainties in structural dynamics. International Journal for Numerical Methods in Engineering, 89:241-268, 2012. 
[28] C. Canuto and T. Kozubek. A fictitious domain approach to the numerical solution of pdes in stochastic domains. Numerische Mathematik, 107(2):257-293, 2007.

[29] A. Nouy, A. Clément, F. Schoefs, and N. Moës. An eXtended Stochastic Finite Element Method for solving stochastic partial differential equations on random domains. Computer Methods in Applied Mechanics and Engineering, 197(51-52):4663-4682, 2008.

[30] A. Nouy, M. Chevreuil, and E. Safatly. Fictitious domain method and separated representations for the solution of boundary value problems on uncertain parameterized domains. Computer Methods in Applied Mechanics and Engineering, 200:3066-3082, 2011.

[31] B.I. Wohlmuth. Discretization methods and iterative solvers based on domain decomposition. 2001.

[32] D. Xiu and D. M. Tartakovsky. Numerical methods for differential equations in random domains. SIAM J. Sci. Comput., 28(3):1167-1185, 2006.

[33] U. Bnerjee I. Babuška and J.E. Osborn. Survey of meshless and generalized finite element methods: A unified approach. Acta Numerica, 12:1-125, 2003.

[34] J. Xu. Iterative methods by space decomposition and subspace correction. SIAM Review, 34(4):581-613, 1992.

[35] T. G. Kolda and B. W. Bader. Tensor decompositions and applications. SIAM Review, 51(3):455-500, September 2009.

[36] F. Chinesta, P. Ladevèze, and E. Cueto. A short review in model order reduction based on proper generalized decomposition. Archives of Computational Methods in Engineering, 18(4):395-404, 2011. 